Research Article

Open Access

Nicolò Zava*

\title{
On a notion of entropy in coarse geometry
}

https://doi.org/10.1515/taa-2019-0005

Received March 18, 2019; accepted July 31, 2019

Abstract: The notion of entropy appears in many branches of mathematics. In each setting (e.g., probability spaces, sets, topological spaces) entropy is a non-negative real-valued function measuring the randomness and disorder that a self-morphism creates. In this paper we propose a notion of entropy, called coarse entropy, in coarse geometry, which is the study of large-scale properties of spaces. Coarse entropy is defined on every bornologous self-map of a locally finite quasi-coarse space (a recent generalisation of the notion of coarse space, introduced by Roe). In this paper we describe this new concept, providing basic properties, examples and comparisons with other entropies, in particular with the algebraic entropy of endomorphisms of monoids.

Keywords: coarse space, quasi-coarse space, coarse entropy, algebraic entropy, growth of metric spaces, coarse equivalence

MSC: 20K30, 37B40, 54H20, 54C70, 54A99, 54E15, 54E99

\section{Introduction}

In 1865 Clausius defined the notion of entropy in physics, but it was only in 1948 that Shannon ([39]) introduced it in mathematics, and, more precisely, in information theory. Inspired by that concept, several other entropies have been introduced in mathematics so far. For example, let us cite Kolmogorov ([25]) and Sinai's ([40]) measure theoretic entropy in ergodic theory, and Adler, Konheim and McAndrew's topological entropy ([1]). Other notions of topological entropy were given by Bowen ([4]) and Hood ([22]). In algebraic dynamics, we can cite the work of Adler, Konheim and MacAndrew ([1]), the entropy defined by Weiss in [43], and the one introduced by Peters ([31], and deeply studied in [12]), that was then generalised in [10] for endomorphisms of abelian groups (we refer to [8] for the definition in the non-abelian case, while to [11] for the extension to endomorphisms of semigroups). Later, Peters in [32] gave an extension of the algebraic entropy defined in [31] for topological automorphisms of locally compact abelian groups. This definition was generalised by Virili ([42]) to all endomorphisms of locally compact abelian groups. This definition can be found in [8] also for non-abelian groups. We finally mention the paper [11], where a unifying approach to several notions of entropy is provided by using normed semigroups. More recently the entropy generated by actions of amenable semigroups and groups have been studied. In particular, Ornstein and Weiss introduced topological and measure entropy of amenable group actions ([30]), whose approach was extended to the case of actions of amenable cancellative semigroups by Ceccherini-Silberstein, Coornaert and Krieger ([5]), Hofmann and Stoyanov studied topological entropy of locally compact semigroup actions on metric spaces ([21]), and Dikranjan, Fornasiero and Giordano Bruno in [6] defined and discussed the algebraic entropy of an action of an amenable cancellative semigroup on an abelian group.

The aim of this paper is to introduce a notion of entropy in coarse geometry. Coarse geometry, also known as large-scale geometry, is the study of large-scale properties of spaces. Intuitively, two spaces are considered equivalent if they look roughly alike for an observer whose point of view is getting further and further

^Corresponding Author: Nicolò Zava: Department of Mathematics, Computer Science and Physics, University of Udine, Via Delle Scienze 206, 33100 Udine, Italy, E-mail: nicolo.zava@gmail.com 
away. While some ideas already appeared for example in Mostow's rigidity theorem, and in Švarc, Milnor, and Wolf's works, a great impetus was provided by Gromov's polynomial growth theorem ([19]). This theory found then many applications in different areas of mathematics, such as geometric group theory, geometric topology, coarse Baum-Connes conjecture, and Novikov conjecture. See the book of Nowak and Yu ([29]) for a comprehensive historical introduction to the topic, and [20] for a more detailed discussion on applications to geometric group theory. Coarse geometry was initially developed for metric spaces. However, some structures, capturing and generalising the large-scale behaviour of metric spaces, appeared in literature, as a large-scale counterpart of uniform spaces ([23]). Roe introduced coarse spaces ([38]) following Weil's approach via entourages, Protasov and Banakh ([35]) defined balleans, generalising the metric ball structure, and then Protasov ([34]), and Dydak and Hoffland ([17]) independently mimed Tukey approach via covers by proposing asymptotic proximities and large-scale structures, respectively. The equivalences between these approaches are discussed in [37] and [13]. Recently, in [44], the basic notions of coarse geometry were extended to asymmetric spaces, such as quasi-metric spaces and monoids, by introducing quasi-coarse spaces. The construction there given is the large-scale counterpart of the classical notion of quasi-uniform space (see the monograph [24] and the survey [26] for a wide introduction to this topic). The morphisms between both coarse and quasi-coarse spaces that are considered are called bornologous maps.

In this paper we introduce coarse entropy $\mathrm{h}_{c}(f)$ (Definition 2.1), defined on the class of bornologous selfmaps $f$ (Definition 1.4) of those quasi-coarse spaces (Definition 1.1) that are locally finite, i.e., whose balls are finite. Its definition involves a limit superior, which is not a limit even when we consider the identity map (Example 2.3), and two supremum operations. The first one is among all entourages, while the second one is among all base points. As for the first one, we show that it is enough to consider just a base of the quasi-coarse structure, while in the second one we just need to evaluate the points that are maximal in the reachability preorder (Proposition 2.2). We then prove basic properties of coarse entropy, such as the weak logarithmic law (Proposition 3.3), how it behaves while taking products (weak addition theorem) and coproducts (Theorem 3.5), and the monotonicity under taking invariant coarse subspaces (Corollary 3.8).

As for conjugation invariance results, that play an important role in the theory of entropy, we have to distinguish two cases. If we consider asymorphisms as isomorphisms, then we obtain a conjugation invariance result for all bornologous self-maps of locally finite quasi-coarse spaces (Corollary 3.9(a)). If, otherwise, we consider Sym-coarse equivalences, a generalisation of the notion of coarse equivalence to the realm of quasicoarse spaces, as isomorphisms then we prove the desired result for quasi-coarse spaces with bounded geometry and for some particular bornologous self-maps (Corollary 3.9(b)). Note that asymorphisms are the isomorphisms of the category QCoarse of quasi-coarse spaces and bornologous maps between them, while Symcoarse equivalences are the isomorphisms of its quotient category QCoarse $/ \sim_{\text {sym }}$ under the Sym-closeness relation (see [44]).

Among the maps for which the conjugation invariance result for Sym-coarse equivalences holds, of a particular interest is the identity map. We can rewrite this specific case as follows: if $X$ and $Y$ are two Sym-coarsely equivalent quasi-coarse spaces with bounded geometry, then $\mathrm{h}_{c}\left(i d_{X}\right)=\mathrm{h}_{c}\left(i d_{Y}\right)$ (Corollary 4.1). Moreover, for every locally finite quasi-coarse space $X, \mathrm{~h}_{c}\left(i d_{X}\right) \in\{0, \infty\}$ (Theorem 4.4). Those results play a key role in connecting the coarse entropy with the growth of metric spaces ([2]), extending the known results for finitely generated groups $([8,20])$. In particular, we show that, for a monogenic metric space $X$, if $Y$ is a bounded geometry skeleton of $X$, the coarse entropy of the identity map of $Y$ can tell whether $X$ has subexponential or exponential growth type (Theorem 4.9).

On monoids and groups, of a particular interest are functorial quasi-coarse structures. In [14], inspired by the notion of functorial topologies ([7]), functorial coarse structures on groups are defined. Informally, a functorial coarse structure $\mathrm{F}$ on groups associates to every group $G$ a group coarse structure $\mathrm{F}(G)$ on $G$ in such a way that, for every group homomorphism $f: G \rightarrow H, f:(G, \mathrm{~F}(G)) \rightarrow(H, \mathrm{~F}(H))$ is bornologous. In [15] functorial coarse structures on topological groups are defined and studied. This notion can be readily extended to functorial quasi-coarse structures on monoids. Among these functorial quasi-coarse structures on monoids, the so-called monoid-quasi-coarse structure (Example 1.2(b)) plays an important role. Moreover, every monoid endowed with its monoid-quasi-coarse structure has bounded geometry. Hence it is natural to compare in this setting the coarse entropy with the algebraic entropy (in the definition provided in [10]). It 
turns out that the algebraic entropy provides an upper bound to the coarse entropy, while they coincide if the endomorphism is surjective (Theorem 5.2). We also provide examples in which the two notions differ for nonsurjective endomorphisms (Examples 5.3 and 5.4). Thanks to this result and using the Pontryagin duality, we are able to connect the coarse entropy with the topological entropy and the measure entropy in particular cases (Corollaries 5.10 and 5.13).

The paper is organised as follows. In Section 1 we recall the needed background from coarse geometry, including basic definitions (e.g., quasi-coarse and coarse spaces, morphisms, local finiteness), results and examples. Section 2 is devoted to introducing the coarse entropy, discussing thoughtfully the definition and providing the first non-trivial examples, i.e., left shifts of monoids and groups (Example 2.6). In Section 3 we collect the basic properties of the coarse entropy, such as the logarithmic law, theorems involving products and coproducts, monotonicity under taking invariant subspaces and conjugation invariance results. The focus of Section 4 is discussing the coarse entropy of the identity, providing also connections with the growth of metric spaces. Finally, Section 5 is dedicated to describing connections with other known entropies, such as the algebraic (\$5.1), the topological and the measure entropies (§5.2). Moreover, the coarse entropy of some group endomorphisms is computed in $\S 5.1$.

\section{Background on large-scale geometry}

In this section we recall some notions of the classical large-scale geometry. We will give them in a more general setting, namely, the one of quasi-coarse spaces. Quasi-coarse spaces were recently introduced in [44] as a large-scale counterpart of quasi-uniform spaces in order to encode the large-scale properties of asymmetric spaces, e.g., quasi-metric spaces and monoids.

Definition 1.1 ([38, 44]). Let $X$ be a set. A family $\mathcal{E} \subseteq \mathcal{P}(X \times X)$ is a quasi-coarse structure on $X$ if it satisfies the following properties:

(a) $\Delta_{X}=\{(x, x) \mid x \in X\} \in \mathcal{E}$;

(b) $\mathcal{E}$ is an ideal (i.e., closed under taking subsets and finite unions);

(c) for every $E, F \in \mathcal{E}$, the composite of $E$ with $F$ belongs to $\mathcal{E}$ (i.e., $E \circ F=\{(x, z) \mid \exists y \in X:(x, y) \in E,(y, z) \in$ $F\} \in \mathcal{E})$.

The pair $(X, \varepsilon)$ is a quasi-coarse space. An element $E$ of $\varepsilon$ is called entourage.

A quasi-coarse structure $\mathcal{E}$ is a coarse structure if, for every $E \in \mathcal{E}, E^{-1}=\{(x, y) \mid(y, x) \in E\} \in \mathcal{E}$. In that case, the pair $(X, \varepsilon)$ is a coarse space.

The entourages of a quasi-coarse space $(X, \varepsilon)$ can be seen as generalised radii as follows: for every $E \in \mathcal{E}$, and $x \in X$, we call the subset $E[x]=\{y \in X \mid(x, y) \in E\}$ as the ball centred in $x$ with radius $E$. Moreover, if $A \subseteq X$, then we define $E[A]=\bigcup_{a \in A} E[a]$. If $x \in X$, a subset $B$ of $X$ is bounded from $x$ if there exists an entourage $E \in \mathcal{E}$ such that $B \subseteq E[x]$.

Let $X$ be a set. A family $\mathcal{F} \subseteq \mathcal{P}(X \times X)$ is a base for the quasi-coarse structure $\mathcal{E}$ if $\{E \subseteq X \times X \mid \exists F \in \mathcal{F}$ : $E \subseteq F\}=\mathcal{E}$.

Let us present two main examples of quasi-coarse spaces.

Example 1.2 ([44]). (a) Let $X$ be a set and $d$ be an extended pseudo-quasi-metric on $X$, i.e., $d: X \times X \rightarrow \mathbb{R}_{\geq 0} \cup$ $\{\infty\}$ satisfies the following properties:

- $d(x, x)=0$, for every $x \in X$;

- $d(x, y) \leq d(x, z)+d(z, y)$, for every $x, y, z \in X$ (triangle inequality).

As usual, we adopt the convention that $\infty+a=a+\infty=\infty$ and $a \leq \infty$ for all $a \in \mathbb{R}_{\geq 0} \cup\{\infty\}$. For the sake of simplicity, we refer to $(X, d)$ as a quasi-metric space. 
For every $R>0$, we define a particular subset of the square $X \times X$, called the strip of width $R$, as follows:

$$
S_{R}=\bigcup_{x \in X}(\{x\} \times B(x, R)) \subseteq X \times X,
$$

where $B(x, R)$ denotes the closed ball centred in $x$ with radius $R$. Then the family $\left\{S_{R} \mid R>0\right\}$ is a base of the so-called metric quasi-coarse structure $\varepsilon_{d}$.

A metric quasi-coarse structure $\varepsilon_{d}$ is a coarse structure if the extended-pseudo-quasi-metric $d$ satisfies also the symmetry property: $d(x, y)=d(y, x)$, for every $x, y \in X$. However, there are non-symmetric quasi-metric spaces inducing coarse structures, as shown in [44].

(b) Let $M$ be a monoid. An ideal J of subsets of $M$ is a monoid ideal if, for every $K, L \in \mathcal{J}, K L=\{k l \mid k \in K, l \in$ $L\} \in \mathcal{J}$. Let $\mathcal{J}$ be a monoid ideal on $M$. For every $K \in \mathcal{J}$, define the strip of width $K$ as the subset

$$
E_{K}=M(\{e\} \times K)=\bigcup_{x \in M}(\{x\} \times x K) \subseteq M \times M .
$$

Then the family $\left\{E_{K} \mid K \in \mathcal{J}\right\}$ is a base for the quasi-coarse structure $\varepsilon_{\mathcal{J}}$, called the J-quasi-coarse structure. For example, if $M$ is a monoid, the family $[M]^{<\omega}=\{K \subseteq M|| K \mid<\omega\}$ is a monoid ideal, and $\mathcal{E}_{\mathcal{J}}$ is called finitary-quasi-coarse structure. For its importance, in this paper we call it monoid-quasi-coarse structure and it is denoted by $\mathcal{E}_{M}$.

Note that, for every $K \in \mathcal{J}$ and $x \in M, E_{K}[x]=x K$.

If $M$ is a group, then a monoid ideal $\mathcal{J}$ on $M$ is called group ideal (see, for example, [37]) if, for every $K \in \mathcal{J}, K^{-1}=\left\{k^{-1} \mid k \in K\right\} \in \mathcal{J}$. If $\mathcal{J}$ is a group ideal, then $\mathcal{E}_{\mathcal{J}}$ is a coarse structure. This construction can be also found in [27]. For example, for every group $G,[G]^{<\omega}$ is a group ideal, inducing the group-coarse structure. Let us present an important example of group ideal on topological groups. If $G$ is a topological group, then the family $\mathfrak{r} C(G)$ of all relatively compact subsets of $G$ is a group ideal and $\mathcal{E}_{\mathfrak{r e}(G)}$ is called compact-group-coarse structure. The functorial properties of the compact-group-coarse structure have been widely studied in [14] and [15].

Remark 1.3. Let $M$ be a finitely generated monoid and $\Sigma$ be a finite generating set of $M$. Then $M$ can be endowed with its word quasi-metric $d_{\Sigma}: M \times M \rightarrow \mathbb{R}_{\geq 0} \cup\{\infty\}$ associated to $\Sigma$ defined as follows: for every $x, y \in M$,

$$
d_{\Sigma}(x, y)=\left\{\begin{array}{lr}
0 & \text { if } x=y, \\
\inf \left\{n \in \mathbb{N} \mid \exists \sigma_{1}, \ldots, \sigma_{n} \in \Sigma: y=x \sigma_{1} \cdots \sigma_{n}\right\} & \text { otherwise }
\end{array}\right.
$$

(where $\inf \emptyset=\infty$ ). In [44] was noted that, if $\Sigma$ and $\Delta$ are two finite generating sets of the finitely generated monoid $M$, then $\varepsilon_{d_{\Sigma}}=\varepsilon_{d_{\Delta}}=\varepsilon_{M}$.

Let now $M$ be a finitely generated group, and $\Sigma$ be a finite symmetric (i.e., $\Sigma^{-1}=\Sigma$ ) generating set. Then the map $d_{\Sigma}: M \times M \rightarrow \mathbb{R}_{\geq 0}$ defined as in (2) is a left-invariant metric on $G$ (i.e., for every $g, h, k \in G, d_{\Sigma}(k g, k h)=$ $d_{\Sigma}(g, h)$ ), called word metric associated to $\Sigma$. Note that $d_{\Sigma}$ induces the discrete topology, and then $\varepsilon_{d_{\Sigma}}=\varepsilon_{M}=$ $\mathcal{E}_{\mathfrak{r} \mathcal{C}(M)}$. For more details about the word metric of finitely generated groups, see, for example, [20].

If $(X, \mathcal{E})$ is a quasi-coarse space and $Y$ is a subset of $X$, then $Y$ can be endowed with the subspace quasi-coarse structure $\left.\mathcal{E}\right|_{Y}=\{E \cap(Y \times Y) \mid E \in \mathcal{E}\}$.

An important large-scale notion is the one of connectedness. Let $(X, \varepsilon)$ be a quasi-coarse space and $x, y \in$ $X$. We define a preorder $\downarrow$, called reachability preorder on $X$ induced by $\mathcal{E}$ as follows: $x \downarrow y$ if $\{(x, y)\} \in \mathcal{E}$ (equivalently, if there exists $E \in \mathcal{E}$ such that $y$ is contained in the ball centred in $x$ with radius $E$ ). We call an element $x \in X$ a maximum if it is a maximum in the preorder $\downarrow$ (i.e., for every $y \in X, x \downarrow y$ ). A quasi-coarse space is connected if every point is a maximum or, equivalently, if $\bigcup \mathcal{E}=X \times X$. For example, for a monoid endowed with its finitary-quasi-coarse structure, the identity is a maximum. Note that, for a coarse space $X$, there exists a maximum if and only if $X$ is connected.

Let $(X, \mathcal{E})$ be a quasi-coarse space. If $x \in X$, define $Q_{X}^{\downarrow}(x)=\{y \in X \mid\{(x, y)\} \in \mathcal{E}\}$ and $Q_{X}^{\uparrow}(x)=\{y \in X \mid$ $\{(y, x)\} \in \mathcal{E}\}$. If $A \subseteq X, Q_{X}^{\downarrow}(A)=\bigcup_{x \in A} Q_{X}^{\downarrow}(x)$ and, similarly, $Q_{X}^{\uparrow}(A)=\bigcup_{x \in A} Q_{X}^{\uparrow}(x)$. Then a point $x \in X$ is a 
maximum if and only if $Q_{X}^{\downarrow}(x)=X$. If $X$ is a coarse space, then, for every $A \subseteq X, Q_{X}^{\downarrow}(A)=Q_{X}^{\uparrow}(A)$ and we will denote it by $Q_{X}(A)$.

A quasi-coarse space $(X, \mathcal{E})$ is locally finite if, for every $E \in \mathcal{E}$ and $x \in X, E[x]$ is finite. Moreover, it has bounded geometry if there exists a map $\delta: \mathcal{E} \rightarrow \mathbb{N}$ such that, for every $E \in \mathcal{E},|E[x]| \leq \delta(E)$. For example, every monoid endowed with the finitary-quasi-coarse structure has bounded geometry.

In order to define the morphisms, we need the notion of Sym-closeness. Let $f, g: S \rightarrow(X, \varepsilon)$ be two maps from a set to a quasi-coarse space. Then $f$ and $g$ are Sym-close (and we denote it by $f \sim_{\text {Sym }} g$ ) if there exists $E=E^{-1} \in \mathcal{E}$ such that, for every $x \in S,(f(x), g(x)) \in E$.

Definition 1.4. A map $f:\left(X, \mathcal{E}_{X}\right) \rightarrow\left(Y, \mathcal{E}_{Y}\right)$ between quasi-coarse spaces is

- bornologous if, for every $E \in \mathcal{E}_{X},(f \times f)(E) \in \mathcal{E}_{Y}$;

- a coarse embedding if it is bornologous and, for every $E \in \mathcal{E}_{Y},(f \times f)^{-1}(E) \in \mathcal{E}_{X}$;

- large-scale injective if $R_{f}=(f \times f)^{-1}\left(\Delta_{Y}\right) \in \mathcal{E}_{X}$;

- large-scale surjective if there exists $E=E^{-1} \in \mathcal{E}_{Y}$ such that $E[f(X)]=Y$ (we also say that $f(X)$ is Sym-large in $Y$ );

- an asymorphism if $f$ is bijective and both $f$ and $f^{-1}$ are bornologous;

- a Sym-coarse equivalence if one of the following equivalent conditions holds:

- $f$ is a large-scale surjective coarse embedding,

- $f$ is bornologous and there exists another bornologous map $g: Y \rightarrow X$ (called Sym-coarse inverse) such that $f \circ g \sim_{\text {Sym }} i d_{Y}$ and $g \circ f \sim_{\text {Sym }} i d_{X}$.

Let us add some remarks concerning the morphisms introduced in Definition 1.4.

Remark 1.5. (a) We say that two quasi-coarse spaces are Sym-coarsely equivalent if there exists a Sym-coarse equivalence between them. In [44] there is another characterisation of Sym-coarsely equivalent quasicoarse spaces. Namely, two quasi-coarse spaces $X$ and $Y$ are Sym-coarsely equivalent if and only if there exist two Sym-large subspaces $X^{\prime} \subseteq X$ and $Y^{\prime} \subseteq Y$ such that $X^{\prime}$ and $Y^{\prime}$ are asymorphic.

(b) Let $f: M \rightarrow N$ be a homomorphism between two monoids (i.e., for every $x, y \in M, f(x y)=f(x) f(y)$ and $\left.f\left(e_{M}\right)=e_{N}\right)$. Then $f$ is bornologous provided that both monoids are endowed with their monoid-quasicoarse structures. A sharper computation is useful: for every $K \in[M]^{<\omega}$,

$$
(f \times f)\left(E_{K}\right)=E_{f(K)} \cap(f(M) \times f(M)) \subseteq E_{f(K)} .
$$

In the case of groups, this "functorial property" is defined and widely studied in [14].

Let us now introduce an important class of quasi-coarse spaces. A quasi-coarse space $(X, \mathcal{E})$ is monogenic if there exists $E \in \mathcal{E}$ such that $\left\{E^{n} \mid n \in \mathbb{N}\right\}$ is a base of $\mathcal{E}$, where $E^{n}$ is the composite of $n$ copies of $E$. In particular, every monogenic quasi-coarse space is metrisable since it has a countable base (see [44]). For example, every finitely generated monoid is monogenic if it is endowed with its word quasi-metric (see Remark 1.3). In fact, let $M$ be a monoid generated by the finite subset $e \in \Sigma$. Then $\varepsilon_{M}$ is generated by the entourage $E_{\Sigma}$. The notion of monogenicity is invariant under Sym-coarse equivalence, as Lemma 1.6 states.

Lemma 1.6. Let $\left(X, \mathcal{E}_{X}\right)$ and $\left(Y, \varepsilon_{Y}\right)$ be two Sym-coarsely equivalent quasi-coarse spaces. Then $X$ is monogenic if and only if $Y$ is monogenic.

Proof. Since monogenicity is trivially preserved under taking asymorphisms, according to Remark 1.5(a), it is enough to consider the case when $Y$ is a Sym-large subspace of $X$ (and $\mathcal{E}_{Y}=\left.\mathcal{E}_{X}\right|_{Y}$ ). Then fix a symmetric entourage $M=M^{-1} \in \mathcal{E}_{X}$ such that $M[Y]=X$.

Suppose that $X$ is monogenic and $E \in \mathcal{E}_{X}$ is an entourage such that $\left\{E^{n} \mid n \in \mathbb{N}\right\}$ is a base of $\mathcal{E}_{X}$. Let $F \in$ $\left.\mathcal{E}_{X}\right|_{Y}$. Then there exists $n_{F} \in \mathbb{N}$ such that $F \subseteq E^{n_{F}}$. Let $(x, y) \in F$. Then there exists $z_{0}=x, z_{1}, \ldots, z_{n}=y \in X$ such that $\left(z_{i}, z_{i+1}\right) \in E$, for every $i=0, \ldots, n-1$. Moreover, for every $i=1, \ldots, n-1$, there exists $z_{i}^{\prime} \in Y$ such that $\left(z_{i}, z_{i}^{\prime}\right) \in M$. Then, if we define $z_{0}^{\prime}=x$ and $z_{n}^{\prime}=y$, for every $i=0, \ldots, n-1,\left(z_{i}^{\prime}, z_{i+1}^{\prime}\right) \in(M \circ E \circ M) \cap(Y \times Y)$. Hence $\left\{((M \circ E \circ M) \cap(Y \times Y))^{n} \mid n \in \mathbb{N}\right\}$ is a base of $\varepsilon_{Y}$. 
Conversely, suppose that $Y$ is monogenic and $\left\{E^{n} \mid n \in \mathbb{N}\right\}$ is a base of $\mathcal{E}_{Y}$, for some $E \in \mathcal{E}_{Y}$. By using a similar argument, it is easy to show that $\left\{(M \circ E \circ M)^{n} \mid n \in \mathbb{N}\right\}$ is a base of $\varepsilon_{X}$.

In particular, Lemma 1.6 implies that a Sym-large subspace of a quasi-coarse space $X$ is monogenic if and only if $X$ itself is monogenic. Note that monogenicity is not inherited by arbitrary subspaces. For example, while $\mathbb{N}$, endowed with the usual euclidean metric coarse structure, is monogenic, the subspace $A=\left\{n^{2} \mid n \in \mathbb{N}\right\} \subseteq \mathbb{N}$ is not monogenic.

Before concluding this section, we introduce two categorical constructions. See [44] for a more comprehensive treatment of the category of quasi-coarse spaces.

Let $\left(X, \varepsilon_{X}\right)$ and $\left(Y, \varepsilon_{Y}\right)$ be two quasi-coarse spaces. Let $p_{X}: X \times Y \rightarrow X$ and $p_{Y}: X \times Y \rightarrow Y$ be the canonical projections. For the sake of simplicity, if $E \in \mathcal{E}_{X}$ and $F \in \mathcal{E}_{Y}$, denote by $E \times F=\left(p_{X} \times p_{X}\right)^{-1}(E) \cap\left(p_{Y} \times p_{Y}\right)^{-1}(F)$. Then the product quasi-coarse structure on $X \times Y$ is $\mathcal{E}_{X} \times \mathcal{E}_{Y}$, which is generated by the base $\left\{E \times F \mid E \in \mathcal{E}_{X}, F \in\right.$ $\left.\mathcal{E}_{Y}\right\}$. If $f: X \rightarrow X$ and $g: Y \rightarrow Y$ are two self-maps, denote by $f \times g: X \times Y \rightarrow X \times Y$ the self-map such that, for every $(x, y) \in X \times Y, f \times g:(x, y) \mapsto(f(x), g(x))$. Moreover, $f \times g$ is bornologous if and only if both $f$ and $g$ are bornologous.

Let $\left\{\left(X_{k}, \varepsilon_{k}\right)\right\}_{k \in I}$ be a family of quasi-coarse spaces and $X=\bigsqcup_{k} X_{k}$ be the disjoint union of their supports. For every $k \in I$, denote by $i_{k}: X_{k} \rightarrow X$ the canonical inclusion. For every $J \in[I]^{<\omega}$ and $\varphi: J \rightarrow \bigcup_{k \in I} \varepsilon_{k}$ such that, for every $k \in J, \varphi(k) \in \mathcal{E}_{k}$, we define

$$
E_{J, \varphi}=\Delta_{X} \cup\left(\bigcup_{j \in J}\left(i_{j} \times i_{j}\right)(\varphi(j))\right) .
$$

Then the coproduct quasi-coarse structure $\bigoplus_{k} \varepsilon_{k}$ on $X$ is defined by the base consisting of the elements $E_{J, \varphi}$, where $J$ and $\varphi$ are as above. If, for every $k \in I, f_{k}: X_{k} \rightarrow X_{k}$ is a self-map, define $\bigoplus_{k} f_{k}: \bigsqcup_{k} X_{k} \rightarrow \bigsqcup_{k} X_{k}$ as follows: for every $i_{j}(x) \in \bigsqcup_{k} X_{k}, \bigoplus_{k} f_{k}\left(i_{j}(x)\right)=i_{j}\left(f_{j}(x)\right)$. Again, $\bigoplus_{k} f_{k}$ is bornologous if and only if $f_{k}$ is bornologous for every $k \in I$.

\section{Definition of coarse entropy}

Let $(X, \varepsilon)$ be a quasi-coarse space and $f: X \rightarrow X$ be a bornologous self map. Then, for every $x \in X$ and $E \in \mathcal{E}$, define the following families of subsets recursively as follows:

$$
\left\{\begin{array}{l}
\mathrm{T}_{1}(f, x, E)=E[x], \\
\mathrm{T}_{n+1}(f, x, E)=\left(f^{n} \times f^{n}\right)(E)\left[\mathrm{T}_{n}(f, x, E)\right], \quad \text { for every } n \in \mathbb{N} .
\end{array}\right.
$$

More explicitly, for every $n \in \mathbb{N}, x \in X, E \in \mathcal{E}$,

$$
\mathrm{T}_{n+1}(f, x, E)=\left(E \circ(f \times f)(E) \circ \cdots \circ\left(f^{n-1} \times f^{n-1}\right)(E) \circ\left(f^{n} \times f^{n}\right)(E)\right)[x],
$$

which is called the $n+1$-coarse trajectory $\mathrm{T}_{n}(f, x, E)$ with respect to $x$ and $E$. When there is no risk of ambiguity, we will simply call it $n+1$ trajectory. Note that, if $X$ is locally finite, for every bornologous self-map, every trajectory is a finite subset according to (6).

Before defining the coarse entropy, in the notation above, let us focus a bit more on the entourages of the form $\left(f^{n} \times f^{n}\right)(E)$. For every self-map $g: X \rightarrow X$ and every subset $A \subseteq X$, we have that

$$
(g \times g)(E)[A]=g\left(E\left[g^{-1}(A)\right]\right) \subseteq g(X) .
$$

In particular, if $A \cap g(X)=\emptyset$, then $(g \times g)(E)[A]=\emptyset$. Moreover, even if $\Delta_{X} \subseteq E$, (7) implies that the trajectories can decrease.

Note that, for every $n \in \mathbb{N} \backslash\{0\}, x \in x$, and $E \in \mathcal{E}$,

$$
\mathrm{T}_{n}(f, x, E) \subseteq Q_{X}^{\downarrow}(x) \cap f^{n-1}(X) .
$$

In fact, if $y \notin f^{n-1}(X)$, then, for every other $z \in X,(z, y) \notin\left(f^{n-1} \times f^{n-1}\right)(E)$.

Let us define the coarse entropy. 
Definition 2.1. Let $(X, \mathcal{E})$ be a locally finite quasi-coarse space and $f: X \rightarrow X$ be a bornologous self map. If $x \in X, E \in \mathcal{E}$, and $n \in \mathbb{N} \backslash\{0\}$, we define

$$
\begin{gathered}
d_{n}=\frac{\log \left|\mathrm{T}_{n}(f, x, E)\right|}{n}, \quad \mathrm{H}_{c}(f, x, E)=\limsup _{n \rightarrow \infty} d_{n}, \\
\mathrm{H}_{c}^{l o c}(f, x)=\sup _{E \in \mathcal{E}} \mathrm{H}_{c}(f, x, E), \quad \text { and, finally, } \quad \mathrm{h}_{c}(f)=\sup _{x \in X} \mathrm{H}_{c}^{l o c}(f, x) .
\end{gathered}
$$

The value $\mathrm{H}_{c}^{\text {loc }}(f, x)$ and $\mathrm{h}_{c}(f)$ are called the local entropy of $f$ in $x$ and the coarse entropy of $f$, respectively.

Proposition 2.2 discusses more in detail the two supremum operations in (10).

Proposition 2.2. Let $(X, \varepsilon)$ be a locally finite quasi-coarse space and $f: X \rightarrow X$ be a bornologous self-map.

(a) If $E, F \in \mathcal{E}$ are two entourages such that $E \subseteq F$, then, for every $x \in X, \mathrm{H}_{c}(f, x, E) \leq \mathrm{H}_{c}(f, x, F)$. In particular, if $\mathcal{F}$ is a base of $\mathcal{E}$, then $\mathrm{H}_{c}^{l o c}(f, x)=\sup \left\{\mathrm{H}_{c}(f, x, F) \mid F \in \mathcal{F}\right\}$, for every $x \in X$.

(b) If $x \downarrow y$, then $\mathrm{H}_{c}^{l o c}(f, x) \geq \mathrm{H}_{c}^{l o c}(f, y)$. In particular, if $X$ has a maximum $x$, then $\mathrm{h}_{c}(f)=\mathrm{H}_{c}^{l o c}(f, x)$.

Proof. (a) Since, if $E \subseteq F \in \mathcal{E}$, then, for every $n \in \mathbb{N}$, $\left(f^{n} \times f^{n}\right)(E) \subseteq\left(f^{n} \times f^{n}\right)(F)$, the conclusion is trivial.

(b) Let $E \in \mathcal{E}$. Define $E^{\prime}=\{(x, y) \circ E\} \cup E$ and then, for every $n \in \mathbb{N} \backslash\{0\}$,

$$
\begin{aligned}
\mathrm{T}_{n}\left(f, x, E^{\prime}\right) & =\left(f^{n-1} \times f^{n-1}\right)\left(E^{\prime}\right)\left[\cdots\left[(f \times f)\left(E^{\prime}\right)\left[E^{\prime}[x]\right]\right] \cdots\right] \supseteq \\
& \supseteq\left(f^{n-1} \times f^{n-1}\right)(E)[\cdots[(f \times f)(E)[E[y]]] \cdots]=T_{n}(f, y, E)
\end{aligned}
$$

since $E^{\prime}[x]=(\{(x, y)\} \circ E)[x]=E[y]$, which shows the desired inequality. The second part of the assertion trivially follows.

The reader may wonder if the limit superior in (9) is a limit or not. In Example 2.3 we provide a locally finite metric space (Example 2.3(a)) and a metric space with bounded geometry (Example 2.3(c)) such that, for suitable inputs, the sequence $d_{n}$ defined in (9) has no limit.

Example 2.3. Before introducing the announced examples, let us recall a general construction. Let $X=$ $(V, E)$ be a non-directed graph. A path of length $n \in \mathbb{N} \backslash\{0\}$ connecting two distinct vertices $x, y \in V$ is a family $\left\{\left(x_{i}, x_{i+1}\right)\right\}_{i=0}^{n-1}$ of edges such that $x_{0}=x$ and $x_{n}=y$. The vertex set $V$ can be endowed with the path metric $d$, i.e., for every pair of distinct points $x, y \in V, d(x, y)$ is the length of the shortest path connecting $x$ and $y$ provided that it exists, otherwise it is $\infty$. If $X$ is connected as a graph, then the metric $d$ does not take the value $\infty$. In this example, for the sake of simplicity, we write $X$ for the associated metric space, even though writing $V$ is more correct.

(a) We want to define a non-directed graph $X=(V, E)$, where $V \subseteq \mathbb{N} \times \mathbb{N}$ and a pair $\left\{(m, n),\left(m^{\prime}, n^{\prime}\right)\right\} \in E$, where $(m, n),\left(m^{\prime}, n^{\prime}\right) \in V$, if and only if $\left|m-m^{\prime}\right|=1$. In order to define the set $V$, we need to inductively construct a sequence $\left\{K_{n}\right\}_{n}$ of natural numbers. Let $K_{0}=1$. Suppose that we have defined $K_{0}, \ldots, K_{m-1}$. Then

$$
K_{m}=\left\{\begin{array}{lr}
1 & \text { if } \sum_{i=0}^{m-1} K_{i}>2 m, \\
2^{m}-\sum_{i=0}^{m-1} K_{i} & \text { otherwise. }
\end{array}\right.
$$

Finally $V=\bigcup_{m \in \mathbb{N}}\left(\{m\} \times\left\{0, \ldots, K_{m}\right\}\right)$.

Endow $X$ with its path metric and then with the induced metric coarse structure. In particular, $X$ is locally finite, even though it has not bounded geometry. Let us consider the map $i d_{X}$. Since $X$ is connected, in order to compute its coarse entropy, we can just consider the trajectories centred in $(0,0)$. Thanks to the definition of $X$, there exists a strictly increasing sequence $\left(a_{n}\right)_{n}$ of natural numbers such that

$$
\sum_{i=0}^{a_{n}-1} K_{i} \leq 2 a_{n} \quad \text { and } \quad \sum_{i=0}^{a_{n}} K_{i}=2^{a_{n}} .
$$


Then

$$
\begin{gathered}
\liminf _{n \rightarrow \infty} \frac{\log \left|\mathrm{T}_{n}\left(i d_{X},(0,0), 1\right)\right|}{n} \leq \lim _{n \rightarrow \infty} \frac{\log (2 n+1)}{n}=0, \quad \text { and } \\
\quad \limsup _{n \rightarrow \infty} \frac{\log \left|\mathrm{T}_{n}\left(i d_{X},(0,0), 1\right)\right|}{n} \geq \lim _{n \rightarrow \infty} \frac{\log \left(2^{n}\right)}{n}=\log 2 .
\end{gathered}
$$

Hence the limit does not exist. Moreover, $\mathrm{h}_{c}\left(i d_{X}\right) \geq \log 2$. Actually, we will show that $\mathrm{h}_{c}\left(i d_{X}\right)$ can only take values in $\{0, \infty\}$ (Theorem 4.4), and thus $\mathrm{h}_{c}\left(i d_{X}\right)=\infty$.

(b) Let us slightly modify item (a), by changing the sequence $\left\{K_{n}\right\}_{n}$ in (11) to

$$
K_{m}=\left\{\begin{array}{lr}
1 & \text { if } \sum_{i=0}^{m-1} K_{i}>2 m, \\
m^{m}-\sum_{i=0}^{m-1} K_{i} & \text { otherwise. }
\end{array}\right.
$$

The induced coarse space $X$ is still locally finite. However, it can be easily proved that $\mathrm{H}_{c}\left(i d_{X},(0,0), 1\right)=$ $\infty$.

(c) We want to provide now an example of a metric space with bounded geometry for which the sequence $d_{n}$ does not have a limit even when we consider the identity map.

We will define a non-directed graph $X$ and endow it with its path metric. For every $n \in \mathbb{N}$, denote by $T_{n}$ the complete 3-ary tree of height $n+1$, by $a_{n}$ its root, and by $x_{1}^{n}, \ldots, x_{3^{n}}^{n}$ its leaves. Let $i_{n}$ and $j_{n}$ be the two canonical inclusions of $T_{n}$ in the disjoint union $T_{n} \sqcup T_{n}$. Note that $\left|T_{n}\right|=\sum_{i=0}^{n} 3^{i}$. Consider the smallest equivalence relation $\sim_{n}$ on $T_{n} \sqcup T_{n}$ satisfying $i_{n}\left(x_{i}^{n}\right) \sim_{n} j_{n}\left(x_{i}^{n}\right)$, for every $i=1, \ldots, 3^{n}$, and define $D_{n}=\left(T_{n} \sqcup T_{n}\right) / \sim_{n}$. Moreover, for every $n \in \mathbb{N}$, denote by $L_{n}$ the graph consisting of $n+1$ points $y_{0}^{n}, \ldots, y_{n}^{n}$ with the edges $\left\{y_{i}^{n}, y_{i+1}^{n}\right\}$, for every $i=0, \ldots, n-1$.

Let us now describe the graph $X$ through a limit process. Define $k_{0}^{\prime}=1$ and $X_{0}=L_{1}=L_{k_{0}^{\prime}}$. Then, for every $n \in \mathbb{N}$, let

$$
\begin{gathered}
k_{n+1}=\min \left\{k \in \mathbb{N}|| X_{2 n} \mid+\sum_{i=1}^{k} 3^{i} \geq 2^{\operatorname{diam}\left(X_{2 n}\right)+k}\right\}, \quad \text { and } \quad X_{2 n+1}=\left.\left(X_{2 n} \sqcup D_{k_{n+1}}\right)\right|_{\approx_{n}}, \\
\text { where } \approx n \text { is the finest equivalence relation satisfying } y_{k_{n}^{\prime}}^{k_{n}^{\prime}} \approx_{n} i_{k_{n+1}}\left(a_{k_{n+1}}\right),
\end{gathered}
$$

and

$$
\begin{gathered}
k_{n+1}^{\prime}=\min \left\{k \in \mathbb{N}|| X_{2 n+1} \mid+k \leq 2\left(\operatorname{diam}\left(X_{2 n+1}\right)+k\right)\right\}, \quad \text { and } \quad X_{2 n+2}=\left(X_{2 n+1} \sqcup L_{k_{n+1}^{\prime}}\right) \approx_{\approx_{n}^{\prime}}, \\
\text { where } \approx_{n}^{\prime} \text { is the finest equivalence relation satisfying } j_{k_{n+1}}\left(a_{k_{n+1}}\right) \approx_{n}^{\prime} y_{0}^{k_{n+1}^{\prime}} .
\end{gathered}
$$

For the sake of simplicity, for every $n \in \mathbb{N}$, in (13) and (14) we have identified the points of $D_{k_{n+1}}$ and $L_{k_{n+1}^{\prime}}$ with their images in $X_{2 n} \sqcup D_{k_{n+1}}$ and in $X_{2 n+1} \sqcup L_{k_{n+1}^{\prime}}$, respectively. Then the graph $X$ is the direct limit of the family $\left\{X_{n}\right\}_{n \in \mathbb{N}}$ of finite graphs (with the family of obvious inclusion maps). In Figure 1 a representation of this space is provided. The metric space associated with the graph $X$ has bounded geometry since every vertex has degree at most 4.

Similarly to what we have done for item (a), it is not hard to prove that, in the notation of (9), if $E=E_{1}$ and $x=y_{0}^{1}$, then

$$
\liminf _{n \rightarrow \infty} d_{n}=0<\log 2 \leq \limsup _{n \rightarrow \infty} d_{n} .
$$

Hence the sequence $\left\{d_{n}\right\}_{n}$ has no limit.

In Example 2.3(b) we provided an example of a locally finite coarse space $(X, \mathcal{E})$ for which there exist $x \in X$ and $E \in \mathcal{E}$ with $\mathrm{H}_{c}\left(i d_{X}, x, E\right)=\infty$. Note that, if $\left(Y, \mathcal{E}^{\prime}\right)$ is a quasi-coarse space with bounded geometry, then, for every point $y \in Y$ and every $E^{\prime} \in \mathcal{E}^{\prime}, \mathrm{H}_{c}\left(i d_{Y}, y, E^{\prime}\right)<\infty$. More precisely, if $\delta\left(E^{\prime}\right)$ is a uniform bound to the cardinality of the balls with radius $E^{\prime}$, then

$$
\mathrm{H}_{c}\left(i d_{Y}, y, E^{\prime}\right) \leq \limsup _{n \rightarrow \infty} \frac{\log \left(\delta\left(E^{\prime}\right)^{n-1}\right)}{n}=\log \left(\delta\left(E^{\prime}\right)\right) .
$$




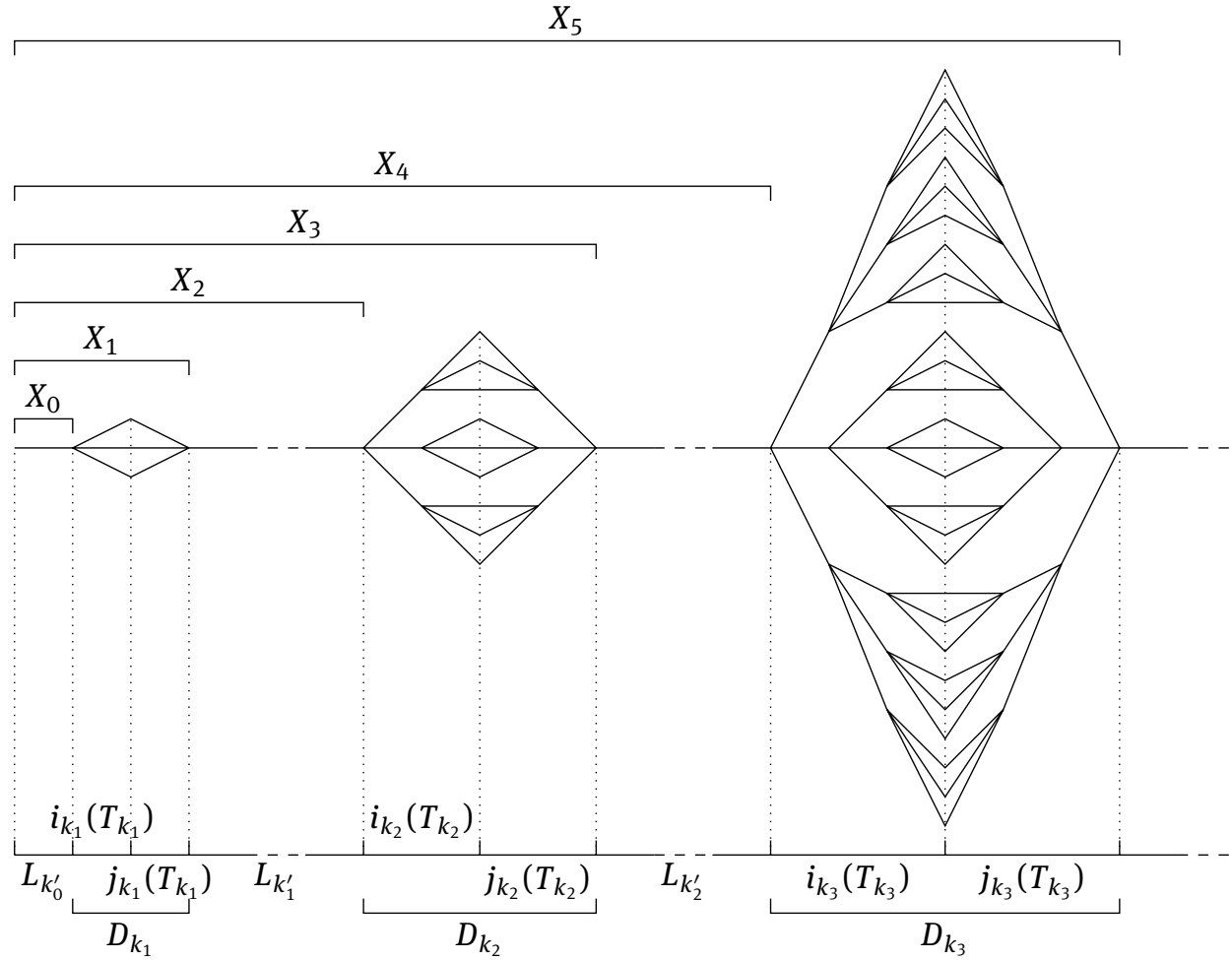

Figure 1: A representation of the graph $X$ defined in Example 2.3(c), underlining the different pieces composing the elements of the family of finite graphs $\left\{X_{n}\right\}_{n}$.

However, the answer to the following question is not known.

Question 2.4. Let $(X, \mathcal{E})$ be a quasi-coarse space with bounded geometry and $f: X \rightarrow X$ be a bornologous self-map. Is it true that, for every $x \in X$ and $E \in \mathcal{E}, \mathrm{H}_{c}(f, x, E)<\infty$ ?

Remark 2.5. Let $(X, \varepsilon)$ be a locally finite quasi-coarse space and $f: X \rightarrow X$ be a bornologous self-map.

(a) Suppose that $f$ is a map such that, for a base $\mathcal{F}$ of $\mathcal{E}$, it satisfies $(f \times f)(F)=F$, for every $F \in \mathcal{F}$. Then, for every $n \in \mathbb{N} \backslash\{0\}, x \in X$ and $F \in \mathcal{F}, \mathrm{T}_{n}(f, x, F)=\mathrm{T}_{n}(i d, x, F)$ and thus, according to Proposition 2.2, $\mathrm{h}_{c}(f)=\mathrm{h}_{c}(i d)$. If, otherwise, there exists a base $\mathcal{H}$ of $\mathcal{E}$ such that $H \supseteq(f \times f)(H) \in \mathcal{H}$, for every $H \in \mathcal{H}$, then $\mathrm{h}_{c}(f) \leq \mathrm{h}_{c}(i d)$.

(b) Let $\mathcal{E}^{\prime}$ be another quasi-coarse structures on $X$ such that $\varepsilon$ is finer than $\mathcal{E}^{\prime}$ (i.e., $\mathcal{E} \subseteq \mathcal{E}^{\prime}$ ). Denote $f_{\mathcal{E}}:(X, \mathcal{E}) \rightarrow(X, \mathcal{E})$ and $\left.f_{\mathcal{E}^{\prime}}:\left(X, \mathcal{E}^{\prime}\right) \rightarrow\left(X, \mathcal{E}^{\prime}\right)\right)$. If $f_{\mathcal{E}^{\prime}}$ is bornologous, then $\mathrm{h}_{c}\left(f_{\mathcal{E}}\right) \leq \mathrm{h}_{c}\left(f_{\mathcal{E}^{\prime}}\right)$ since, for every $x \in X, \mathrm{H}_{c}^{l o c}\left(f_{\varepsilon}, x\right) \leq \mathrm{H}_{c}^{l o c}\left(f_{\mathcal{E}^{\prime}}, x\right)$.

(c) If $E \in \mathcal{E}$, define $E^{\square}=\bigcup_{n \in \mathbb{N}} E^{n}$. The quasi-coarse space $(X, \mathcal{E})$ is called cellular if $E^{\square} \in \mathcal{E}$, for every $E \in \mathcal{E}$. Cellular coarse spaces are precisely those with asymptotic dimension 0 ([37]). If $X$ is cellular, then $\mathrm{h}_{c}(i d)=0$ since the trajectories stabilise. More precisely, for every choice of $E \in \mathcal{E}$, and $x \in X$, the $n$-th trajectory is contained in a subset, namely $E^{\square}[x]$, which is bounded from $x$ and thus finite. Note that a monoid, endowed with the monoid-quasi-coarse structure is cellular if and only if it is locally finite (i.e., for every $K \in[M]^{<\omega}$, the submonoid generated by $K$ is still finite).

Example 2.6. Let $M$ be a monoid endowed with the monoid-quasi-coarse structure and $x \in M$. We want to discuss the entropy of the left shift $f=s_{x}^{\lambda}: y \mapsto x y$. First of all, let us note that, for every $K \in[M]^{<\omega}$,

$$
(f \times f)\left(E_{K}\right) \subseteq E_{K},
$$


which shows, in particular, that $f$ is bornologous. Since the neutral element $e$ is a maximum in $M$, then we just need to consider the trajectories with respect to $e$ (Proposition 2.2).

(a) Suppose that $x$ is an invertible element of $M$. Then $f$ is an asymorphism with inverse $s_{x^{-1}}^{\lambda}$. Moreover, it is easy to see that (15) becomes an equality and thus Remark 2.5(a) implies that $\mathrm{h}_{c}(f)=\mathrm{h}_{c}\left(i d_{M}\right)$. In particular this equality holds if $M$ is a group.

(b) Let $M$ be left-cancellative (i.e., for every $y \in M$, the left shift $s_{y}^{\lambda}$ is injective) and commutative. Split $K=$ $(K \cap f(M)) \cup(K \backslash f(M))$. Note that $(f \times f)\left(E_{K}\right)[K]=(f \times f)\left(E_{K}\right)[K \cap f(M)]$, according to (7). Hence, without loss of generality, we can assume that $K=x F$, for some non-empty $F \in[M]^{<\omega}$. By using induction, (7), and the commutativity of $M$, we can prove that, for every $n \in \mathbb{N}, \mathrm{T}_{n}\left(f, e, E_{K}\right)=n x+n F$, where $n F$ is the family of elements of $M$ that can be written as sum of $n$ elements of $F$. Moreover, since $M$ is left-cancellative, $\left|\mathrm{T}_{n}\left(f, e, E_{K}\right)\right|=|n F|=\left|\mathrm{T}_{n}\left(i d, e, E_{F}\right)\right|=\left|\mathrm{T}_{n}\left(i d, e, E_{K}\right)\right|$. Thus $\mathrm{H}_{c}\left(f, e, E_{K}\right)=\mathrm{H}_{c}\left(i d, e, E_{K}\right)=\mathrm{H}_{c}\left(i d, e, E_{F}\right)$.

(c) Let $M$ be the free monoid generated by two elements $a$ and $b$, and $x=a$. We claim that $\mathrm{h}_{c}(f)=\infty$. Because of Proposition 2.2 we can restrict ourselves to study the base $\left\{E_{B(e, m)} \mid m \in \mathbb{N}\right\}$, where $B(e, m)$, for every $m \in \mathbb{N}$, represents the closed ball centred in $e$ with radius $m$ associated to the word quasi-metric $d_{\{a, b\}}$. If $K=B(e, m)$, we claim that

$$
\mathrm{T}_{n+1}\left(f, e, E_{K}\right)=a^{n+1} B(e,(n+1) m-(n+1))+a^{n} b B(e,(n+1) m-(n+1))=a^{n} B(e, n m+m-n) .
$$

If $n=0$, then (16) is trivial. Suppose now that (16) holds for some $n \in \mathbb{N}$. Then

$$
\begin{aligned}
\mathrm{T}_{n+2}\left(f, e, E_{K}\right) & =\left(\left(f^{n+1} \times f^{n+1}\right)\left(E_{K}\right)\right)\left[\mathrm{T}_{n+1}(f, e, K)\right]= \\
& =\left(\left(f^{n+1} \times f^{n+1}\right)\left(E_{K}\right)\right)\left[a^{n+1} B(e,(n+1) m-(n+1))+a^{n} b B(e,(n+1) m-(n+1))\right]= \\
& =f^{n+1}\left(E_{K}\left[\left(f^{n+1}\right)^{-1}\left(a^{n+1} B(e,(n+1) m-(n+1))+a^{n} b B(e,(n+1) m-(n+1))\right)\right]\right)= \\
& =a^{n+1} B(e,(n+1) m-(n+1)) B(e, m)=a^{n+1} B(e,(n+2) m-(n+1))= \\
& =a^{n+2} B(e,(n+2) m-(n+2))+a^{n+1} b B(e,(n+2) m-(n+2)),
\end{aligned}
$$

which shows (16). Then,

$$
\mathrm{H}_{c}\left(f, e, E_{B(e, m)}\right) \geq \limsup _{n \rightarrow \infty} \frac{\log \left(2^{n(m-1)}\right)}{n} \geq(m-1) \log 2,
$$

and so the claim follows since $m$ can be arbitrarily taken.

\section{Basic properties of the coarse entropy}

Before computing more, less trivial, examples of the coarse entropy (in Section 5 many examples appear, in relation with the algebraic entropy), we focus on proving some standard properties of the coarse entropy.

Proposition 3.1. Let $(X, \mathcal{E})$ be a locally finite quasi-coarse space, $f: X \rightarrow X$ be a bornologous self-map and $x \in X$.

(a) If there exists $F \in \mathcal{E}$ such that $Q_{X}^{\downarrow}(x)=F[x]$, then $\mathrm{H}_{c}^{\text {loc }}(f, x)=0$.

(b) If there exists $E \in \mathcal{E}$ and $n \in \mathbb{N}$ such that $f^{n}(X) \subseteq E[x]$, then $\mathrm{h}_{c}(f)=0$.

(c) If there exists $n \in \mathbb{N}$ such that $x \notin Q_{X}^{\uparrow}\left(f^{n}(X)\right)$, then $\mathrm{H}_{c}^{\text {loc }}(f, x)=0$.

(d) If $x \notin \bigcap_{n} Q_{X}^{\uparrow}\left(f^{n}(X)\right)$, then $\mathrm{H}_{c}^{\text {loc }}(f, x)=0$.

Proof. Items (a) and (b) trivially follow from (8) and from the fact that $X$ is locally finite and thus $F[x]$ and $E[x]$ are finite. Finally, it is enough to show item (c) in order to prove item (d). If $x \notin \mathcal{Q}_{X}^{\uparrow}\left(f^{n}(X)\right)$, then $Q_{X}^{\downarrow}(x) \cap f^{n}(X)=$ $\emptyset$. Hence, (8) implies that, $\mathrm{T}_{n+1}(f, x, E) \subseteq Q_{X}^{\downarrow}(x) \cap f^{n-1}(X)=\emptyset$, for every $E \in \mathcal{E}$. Morevoer, for every $k>n$, $f^{k}(X) \subseteq f^{n}(X)$, and thus $\mathrm{T}_{k}(f, x, E)=\emptyset$. Hence, $\mathrm{H}_{c}(f, x, E)=0$ and $\mathrm{H}_{c}^{l o c}(f, x)=0$.

Let $X$ be a locally finite quasi-coarse space, and $f: X \rightarrow X$ be a bornologous self-map. A subset $Y$ of $X$ is called $f$-invariant if $f(Y) \subseteq Y$. 
Corollary 3.2. Let $(X, \mathcal{E})$ be a locally finite quasi-coarse space and $f: X \rightarrow X$ be a bornologous self-map. The subspace $Y=\bigcap_{n} Q_{X}^{\uparrow}\left(f^{n}(X)\right)$ is $f$-invariant and $\mathrm{h}_{c}(f)=\mathrm{h}_{c}\left(\left.f\right|_{Y}\right)$.

Proof. Let $y \in Y$. Then, for every $n \in \mathbb{N}$, there exists $x_{n} \in X$ such that $\left\{\left(y, f^{n}\left(x_{n}\right)\right)\right\} \in \mathcal{E}$. In particular, since $f$ is bornologous, $\left\{\left(f(y), f^{n+1}\left(x_{n}\right)\right)\right\} \in \mathcal{E}$, which implies that $y \in Q_{X}^{\uparrow}\left(f^{n+1}(X)\right)$. Since the chain $\left\{Q_{X}^{\uparrow}\left(f^{n}(X)\right) \mid n \in \mathbb{N}\right\}$ is decreasing, $f(y) \in \mathcal{Q}_{X}^{\uparrow}(f(X))$ and thus the first statement is proved. The equality follows from Proposition 3.1(d).

Proposition 3.3 (Weak logarithmic law). Let $(X, \mathcal{E})$ be a locally finite quasi-coarse space and $f: X \rightarrow X$ be a bornologous self-map. If $f$ is surjective, for every $k>0, \mathrm{~h}_{c}\left(f^{k}\right) \leq k \cdot \mathrm{h}_{c}(f)$.

Proof. Fix a positive integer $k>0$. Then, for every $n \in \mathbb{N}, x \in X$, and $\Delta_{X} \subseteq E \in \mathcal{E}, \mathrm{T}_{n}\left(f^{k}, x, E\right) \subseteq$ $T_{k n-k+1}(f, x, E)$ since the surjectivity of $f$ implies that $\Delta_{X} \subseteq\left(f^{s} \times f^{s}\right)(E)$, for every $s \in \mathbb{N}$, and so

$$
\begin{aligned}
\mathrm{H}_{c}\left(f^{k}, x, E\right) & =\limsup _{n \rightarrow \infty} \frac{\log \left|\mathrm{T}_{n}\left(f^{k}, x, E\right)\right|}{n} \leq \limsup _{n \rightarrow \infty} \frac{\log \left|\mathrm{T}_{k n-k+1}(f, x, E)\right|}{k n-k+1} \cdot \frac{k n-k+1}{n} \leq \\
& \leq k \mathrm{H}_{c}(f, x, E) \leq k \mathrm{~h}_{c}(f),
\end{aligned}
$$

from which the stated inequality follows.

Question 3.4. Does the opposite inequality in Proposition 3.3 hold?

The next result, Theorem 3.5, states that the coarse entropy behaves as expected in relation with (finite) products and coproducts of quasi-coarse spaces. We consider only finite products since arbitrary products of locally finite quasi-coarse spaces are not necessarily locally finite.

Theorem 3.5. (a) Let $\left(X, \mathcal{E}_{X}\right)$ and $\left(Y, \mathcal{E}_{Y}\right)$ be two locally finite quasi-coarse spaces and $f: X \rightarrow X$ and $g: Y \rightarrow$ $Y$ be two bornologous self-maps. Then $\mathrm{h}_{c}(f \times g)=\mathrm{h}_{c}(f)+\mathrm{h}_{c}(g)$.

(b) Let $\left\{\left(X_{k}, \mathcal{E}_{k}\right)\right\}_{k \in I}$ be a family of locally finite quasi-coarse spaces and, for every $k \in I, f_{k}: X_{k} \rightarrow X_{k}$ be a bornologous self-map. Then $\mathrm{h}_{c}\left(\bigoplus_{k} f_{k}\right)=\sup _{k \in I} \mathrm{~h}_{c}\left(f_{k}\right)$.

Proof. (a) Because of Proposition 2.2(a), we can just evaluate the trajectories $\mathrm{T}_{n}(f \times g,(x, y), E \times F)$, where $n \in \mathbb{N} \backslash\{0\},(x, y) \in X \times Y, E \in \mathcal{E}_{X}$ and $F \in \mathcal{E}_{Y}$. Note that $(E \times F)[(x, y)]=E[x] \times F[y],((f \times g) \times(f \times g))(E \times F)=$ $(f \times f)(E) \times(g \times g)(F)$, and $(f \times g)^{k}=f^{k} \times g^{k}$, for every $k \in \mathbb{N}$. Then

$$
\begin{aligned}
\mathrm{T}_{n}(f \times g,(x, y), E \times F)= & \left((f \times g)^{n-1} \times(f \times g)^{n-1}\right)(E \times F)[\cdots[((f \times g) \times(f \times g)(E \times F))[E \times F[(x, y)]]] \cdots]= \\
= & \left(\left(f^{n-1} \times f^{n-1}\right)(E) \times\left(g^{n-1} \times g^{n-1}\right)(F)\right)[\cdots \\
& \cdots[((f \times f)(E) \times(g \times g)(F))[E \times F[(x, y)]]] \cdots]= \\
= & \mathrm{T}_{n}(f, x, E) \times \mathrm{T}_{n}(g, y, F),
\end{aligned}
$$

and thus

$$
\left|\mathrm{T}_{n}(f \times g,(x, y), E \times F)\right|=\left|\mathrm{T}_{n}(f, x, E)\right| \cdot\left|\mathrm{T}_{n}(g, y, F)\right|,
$$

from which the equality $\mathrm{H}_{c}(f \times g,(x, y), E \times F)=\mathrm{H}_{c}(f, x, E)+\mathrm{H}_{c}(g, y, F)$ and the desired claim follow.

Item (b) trivially follows from the observation that, for every $n \in \mathbb{N} \backslash\{0\}, i_{j}(x) \in \bigsqcup_{k} X_{k}$, and $E_{J, \varphi} \in \bigoplus_{k} \varepsilon_{k}$, defined as in (4), where $J \in[I]^{<\omega}$ and $\varphi: J \rightarrow \bigcup_{k} \varepsilon_{k}$ with the desired properties,

$$
\mathrm{T}_{n}\left(\bigoplus_{k \in I} f_{k}, i_{j}(x), E_{J, \varphi}\right)=i_{j}\left(T_{n}\left(f_{j}, x, F\right)\right), \quad \text { where } \quad F=\left\{\begin{array}{lr}
E_{j} & \text { if } j \in J, \\
\Delta_{X_{j}} & \text { otherwise. }
\end{array}\right.
$$

Conjugation results are particularly important in developing entropies (see also Remark 3.10). The final part of this section is devoted to prove conjugation results for the coarse entropy. 
Lemma 3.6. Let $X$ be a set and $h: X \rightarrow X$ be a self-map. Then:

(a) if $E, F \subseteq X \times X$, then $(h \times h)(E \circ F) \subseteq(h \times h)(E) \circ(h \times h)(F)$;

(b) if $E \subseteq X \times X$ and $x \in X$, then $h(E[x]) \subseteq((h \times h)(E))[h(x)]$.

Proof. (a) Let $(h(x), h(z)) \in(h \times h)(E \circ F)$ such that $(x, z) \in E \circ F$. Then there exists $y \in X$ such that $(x, y) \in E$ and $(y, z) \in F$, which implies $(h(x), h(y))=(h(x), h(y)) \circ(h(y), h(z)) \in(h \times h)(E) \circ(h \times h)(F)$.

(b) Consider an arbitrary $y \in h(E[x])$ and take $z \in h^{-1}(y)$ such that $(x, z) \in E$. Then $(h(x), y)=$ $(h(x), h(z)) \in(h \times h)(E)$, which shows that $y \in((h \times h)(E))[h(x)]$.

Theorem 3.7. Let $(X, \mathcal{E})$ and $\left(Y, \varepsilon_{Y}\right)$ be two locally finite quasi-coarse spaces, $f: X \rightarrow X$ and $g: Y \rightarrow Y$ be two bornologous self-maps, and $h: X \rightarrow Y$ be a bornologous map such that the following diagram commutes:

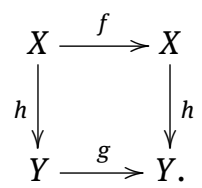

If there exists $K \in \mathbb{N}$ such that $\sup \left\{\left|h^{-1}(y)\right| \mid y \in Y\right\} \leq K$, then $\mathrm{h}_{c}(f) \leq \mathrm{h}_{c}(g)$.

Proof. Let $x \in X, E \subseteq \mathcal{E}_{X}$, and $x \in X$. Then, by applying Lemma 3.6 and the commutativity of (17), for every $n \in \mathbb{N} \backslash\{0\}$, we have

$$
\begin{aligned}
\mathrm{T}_{n}(f, x, E) & \subseteq h^{-1}\left(h\left(T_{n}(f, x, E)\right)\right)=h^{-1}\left(h\left(\left(E \circ(f \times f)(E) \circ \cdots \circ\left(f^{n-1} \times f^{n-1}\right)(E)\right)[x]\right)\right) \subseteq \\
& \subseteq h^{-1}\left((h \times h)\left(E \circ(f \times f)(E) \circ \cdots \circ\left(f^{n-1} \times f^{n-1}\right)(E)\right)[h(x)]\right) \subseteq \\
& \subseteq h^{-1}\left((h \times h)(E) \circ(h \times h)((f \times f)(E)) \circ \cdots \circ\left((h \times h)\left(f^{n-1} \times f^{n-1}\right)(E)\right)[h(x)]\right)= \\
& =h^{-1}\left((h \times h)(E) \circ(g \times g)((h \times h)(E)) \circ \cdots \circ\left(g^{n-1} \times g^{n-1}\right)((h \times h)(E))[h(x)]\right)= \\
& =h^{-1}\left(\mathrm{~T}_{n}(g, h(x),(h \times h)(E))\right) .
\end{aligned}
$$

The computation shows that

$$
\begin{aligned}
\mathrm{H}_{c}(f, x, E) & =\limsup _{n \rightarrow \infty} \frac{\log \left|\mathrm{T}_{n}(f, x, E)\right|}{n} \leq \limsup _{n \rightarrow \infty} \frac{\log \left(K\left|\mathrm{~T}_{n}(g, h(x),(h \times h)(E))\right|\right)}{n}= \\
& =\limsup _{n \rightarrow \infty} \frac{\log K+\log \mid \mathrm{T}_{n}(g, h(x),(h \times h)(E) \mid}{n}=\mathrm{H}_{c}(g, h(x),(h \times h)(E))
\end{aligned}
$$

because of the assumption on the fibers of $h$. Then, since both $E \in \mathcal{E}$ and $x \in X$ are arbitrary, $\mathrm{h}_{c}(f) \leq \mathrm{h}_{c}(g)$.

Corollary 3.8. Let $(X, \mathcal{E})$ and $\left(Y, \varepsilon_{Y}\right)$ be two locally finite quasi-coarse spaces, $f: X \rightarrow X$ and $g: Y \rightarrow Y$ be two bornologous self-maps, and $h: X \rightarrow Y$ be a bornologous map such that the diagram (17) commutes. Suppose, moreover, that one of the following properties holds:

(a) $h$ is injective;

(b) $(X, \mathcal{E})$ has bounded geometry and $h$ is large-scale injective.

Then $\mathrm{h}_{c}(f) \leq \mathrm{h}_{c}(g)$.

Proof. We want to apply Theorem 3.7. If $h$ is injective, then we can set $K=1$. If $h$ is large-scale injective, then $R_{h}=(h \times h)^{-1}\left(\Delta_{Y}\right) \in \mathcal{E}_{X}$. Since $X$ has bounded geometry, there exists $K \in \mathbb{N}$ such that $K \geq\left|R_{h}[x]\right|=\left|h^{-1}(h(x))\right|$, for every $x \in X$.

As an immediate consequence of Corollary 3.8 we have the monotonicity of the coarse entropy under taking invariant subspaces. Let $(X, \mathcal{E})$ be a locally finite quasi-coarse space, $f: X \rightarrow X$ be a bornologous self-map, and $Y$ be a $f$-invariant subset of $X$, then $\mathrm{h}_{c}\left(\left.f\right|_{Y}\right) \leq \mathrm{h}_{c}(f)$. Moreover, the same result implies, in the case of coarse spaces with bounded geometry, the monotonicity of the coarse entropy under taking coarse embeddings. In fact, in the notation of Corollary 3.8, if $h$ is a coarse embedding, then item (b) is fulfilled.

From Corollary 3.8 the following important invariance result trivially follows. 
Corollary 3.9 (Invariance under conjugation). Let $(X, \mathcal{E})$ and $\left(Y, \mathcal{E}_{Y}\right)$ be two locally finite quasi-coarse spaces, $f: X \rightarrow X$ and $g: Y \rightarrow Y$ be two bornologous self-maps, and $h: X \rightarrow Y$ be a map such that the diagram (17) commutes. Suppose, moreover, that one of the following properties holds:

(a) $h$ is an asymorphism;

(b) $X$ and $Y$ have bounded geometry, $h$ is a Sym-coarse equivalence with a Sym-coarse inverse $k: Y \rightarrow X$ such that $f \circ k=k \circ g$.

Then $\mathrm{h}_{c}(f)=\mathrm{h}_{c}(g)$.

Remark 3.10. Let $X$ be a category. We define the category Flow $X$ of flows in $X$. As objects, it has pairs $(X, f)$, where $X \in X$ and $f: X \rightarrow X$ is a morphism of $X$. Moreover, a morphism between two such pairs $(X, f)$ and $(Y, g)$ is a map $h: X \rightarrow Y$ in $X$ such that $h \circ f=g \circ h$. Moreover, $h$ is an isomorphism in Flow $X$ if $h$ is an isomorphism in $X$.

Let QCoarse be the category of quasi-coarse spaces and bornologous maps between them ([44]). We refer to [13] and [45] for a deep investigation of the full subcategory Coarse of QCoarse of all coarse spaces and its quotient category Coarse/, where $\sim$ is the closeness relation, i.e., the Sym-closeness relation restricted to Coarse. Denote by LF-QCoarse the full subcategory of QCoarse of locally finite quasi-coarse spaces. Consider then the category FlowLF-QCoarse. Thanks to Corollary 3.9, if $(X, f)$ and $(Y, g)$ are two isomorphic flows, then $\mathrm{h}_{c}(f)=\mathrm{h}_{c}(g)$. Hence, $\mathrm{h}_{c}$ associates a value in $\mathbb{R}_{\geq 0} \cup\{\infty\}$ to every isomorphism class of flows in FlowLF-QCoarse.

\section{Coarse entropy of the identity and growth of quasi-coarse spaces}

Let us focus on the identity map of a quasi-coarse space. Corollary 3.8 implies that, the coarse entropy of the identity map is an invariant under Sym-coarse equivalence in the realm of quasi-coarse spaces with bounded geometry.

Corollary 4.1. Let $X$ and $Y$ be two Sym-coarsely equivalent quasi-coarse spaces with bounded geometry. Then $\mathrm{h}_{c}\left(i d_{X}\right)=\mathrm{h}_{c}\left(i d_{Y}\right)$.

Proof. Let $f: X \rightarrow Y$ be a Sym-coarse equivalence. Then we can easily apply Corollary 3.8, which implies that $\mathrm{h}_{c}\left(i d_{X}\right) \leq \mathrm{h}_{c}\left(i d_{Y}\right)$. The opposite inequality can be similarly proved.

We want to show that the identity function cannot have arbitrary values. More precisely, if $X$ is a locally finite quasi-coarse space, then $\mathrm{h}_{c}\left(i d_{X}\right) \in\{0, \infty\}$. In order to prove this result, let us recall the following folklore fact.

Lemma 4.2. Let $\left\{a_{n}\right\}_{n}$ and $\left\{b_{n}\right\}_{n}$ be two sequences of non-negative real numbers such that the limit of $\left\{a_{n}\right\}_{n}$ exists and it is strictly positive. Then

$$
\limsup _{n \rightarrow \infty} a_{n} b_{n}=\left(\lim _{n \rightarrow \infty} a_{n}\right)\left(\limsup _{n \rightarrow \infty} b_{n}\right) .
$$

Proof. Since, the two sequences have non-negative values, for every $n \in \mathbb{N}, \sup _{k \geq n}\left(a_{k} b_{k}\right) \leq \sup _{k \geq n} a_{k}$. $\sup _{k \geq n} b_{k}$ and thus the inequality (s) in (18) follows. As for the opposite inequality, fix a value $0<\varepsilon<l=$ $\lim _{n \rightarrow \infty} a_{n}$. Then there exists $N \in \mathbb{N}$ such that, for every $n \geq N, a_{n}>l-\varepsilon$. Thus, for every $k \geq N$,

$$
\sup _{n \geq k} a_{n} b_{n} \geq \sup _{n \geq k}(l-\varepsilon) b_{n}=(l-\varepsilon) \sup _{n \geq k} b_{n}
$$

since $l-\varepsilon>0$, and so

$$
\limsup _{k \rightarrow \infty} a_{k} b_{k} \geq(l-\varepsilon) \limsup _{k \rightarrow \infty} b_{k}=\left(\lim _{n \rightarrow \infty} a_{n}-\varepsilon\right) \limsup _{k \rightarrow \infty} b_{k} .
$$

Since $\varepsilon$ can be arbitrarily taken, (19) implies the desired inequality. 
Lemma 4.3. Let $\left\{a_{n}\right\}_{n}$ be an increasing sequence of positive real numbers. If we denote by $l=\lim \sup _{n \rightarrow \infty} \log a_{n} / n$, then we have $\lim \sup _{n \rightarrow \infty} \log \left(a_{t n}\right) / n=t l$, for every $t \in \mathbb{N} \backslash\{0\}$.

Proof. If $t=1$, then there is nothing to prove. For the sake of simplicity, we prove the result for $t=2$, but the argument can be easily generalised.

Fix $n \geq 1$ and define two sequences $\left\{u_{k}^{n}\right\}_{k \geq n}$ and $\left\{v_{s}^{n}\right\}_{s \geq 2 n-1}$ as follows:

$$
u_{k}^{n}=\frac{\log \left(a_{2 k}\right)}{k}, \quad \text { and } \quad v_{s}^{n}=\frac{\log \left(a_{s}\right)}{\lceil s / 2\rceil}, \quad \text { for every } k \geq n \text { and } s \geq 2 n-1 .
$$

Since, for every $k \geq n, u_{k}^{n}=v_{2 k}^{n}, \sup _{k \geq n} u_{k}^{n} \leq \sup _{s \geq 2 n-1} v_{s}^{n}$. We want to show the opposite inequality. Let then $s \geq 2 n-1$. If $s=2 k$ for some $k \geq n$, then $v_{s}^{n}=u_{k}^{n}$. Otherwise, if $s$ is odd, $v_{s}^{n} \leq u_{(s+1) / 2}^{n}$ and thus

$$
\sup _{k \geq n} u_{k}^{n}=\sup _{s \geq 2 n-1} v_{s}^{n}=\sup _{s \geq 2 n-1} \frac{\log \left(a_{s}\right)}{s} \cdot \frac{s}{\lceil s / 2\rceil} .
$$

Then, since (20) holds for every $n \geq 1$ and the sequence $\{n /\lceil n / 2\rceil\}_{n}$ converges to 2, by applying Lemma 4.2, we obtain that

$$
\limsup _{n \rightarrow \infty} \frac{\log \left(a_{2 n}\right)}{n}=\lim _{n \rightarrow \infty} \frac{n}{\lceil n / 2\rceil} \cdot \limsup _{n \rightarrow \infty} \frac{\log \left(a_{n}\right)}{n}=2 l .
$$

Theorem 4.4. Let $(X, \mathcal{E})$ be a locally finite quasi-coarse space, $f: X \rightarrow X$ be a bornologous self-map, $\Delta_{X} \subseteq E \in$ $\mathcal{E}$, and $x \in X$. Then, $\mathrm{H}_{c}\left(i d, x, E^{k}\right)=k \mathrm{H}_{c}(i d, x, E)$, for every $k \in \mathbb{N}$. In particular, $\mathrm{h}_{c}(i d) \in\{0, \infty\}$.

Proof. Fix a point $x \in X, \Delta_{X} \subseteq E \in \mathcal{E}$, and $k \in \mathbb{N}$. Consider the sequence $a_{n}=\left|\mathrm{T}_{n}(i d, x, E)\right|=\left|E^{n-1}[x]\right|$, for every $n \in \mathbb{N} \backslash\{0\}$. Since $\Delta_{X} \subseteq E,\left\{a_{n}\right\}_{n}$ is increasing and non-negative. Hence, Lemma 4.3 implies the claim since $\mathrm{H}_{c}(i d, x, E)=\lim \sup _{n \rightarrow \infty} \log \left(a_{n}\right) / n$.

From Proposition 2.2(a) and Theorem 4.4 we can deduce an easy corollary.

Corollary 4.5. Let $(X, \mathcal{E})$ be a locally finite quasi-coarse space. Then $\mathrm{h}_{c}\left(i d_{X}\right)=\infty$ if and only if there exists $x \in X$ and $F \in \mathcal{E}$ such that $\mathrm{H}_{c}\left(i d_{X}, x, F\right)>0$. Moreover, if $X$ is monogenic, $E \in \mathcal{E}$ is an entourage such that $\left\{E^{n} \mid n \in \mathbb{N}\right\}$ is a base of $\mathcal{E}$, and $x \in X$. Then:

(a) $\mathrm{H}_{c}^{\text {loc }}(i d, x)=0$ if and only if $\mathrm{H}_{c}(i d, x, E)=0$;

(b) $\mathrm{H}_{c}^{\text {loc }}(i d, x)=\infty$ if and only if $\mathrm{H}_{c}(i d, x, E)>0$.

Let us now connect the coarse entropy with the growth of a metric space. Here we present a generalisation of the approach for metric spaces that can be found in [2]. A quasi-coarse space $(X, \mathcal{E})$ has a bounded geometry skeleton if there exists a subset $Y$ (called bounded geometry skeleton) of $X$ such that

- $\left(Y,\left.\mathcal{E}\right|_{Y}\right)$ has bounded geometry;

- $Y$ is Sym-large in $X$.

In [2], this notion is given only for metric spaces under the name quasi-lattice, and a metric space is said to have coarse bounded geometry if it has a quasi-lattice.

Not every quasi-coarse space has a bounded geometry skeleton, as the following examples show.

Example 4.6. (a) Let $X$ be an infinite set. Fix a point $\bar{x} \in X$ and define the quasi-coarse structure $\varepsilon$ defined by the base $\left\{\Delta_{X} \cup(\{\bar{x}\} \times X)\right\}$. Then $\Delta_{X}$ is the only symmetric entourage in $\mathcal{E}$ and thus $X$ itself is the only Sym-large subset. However $X$ is not locally finite.

(b) Let $X$ be an uncountable group (e.g., take $X=\mathbb{R}$ ) and consider the group ideal $\mathcal{J}=[X]^{<\omega_{1}}=\{Y \subseteq X|| Y \mid<$ $\left.\omega_{1}\right\}$. For the induced group-coarse structure $\mathcal{E}_{\mathcal{J}}$, if a subset $Y$ of $X$ is Sym-large, then it is uncountable. Then there are countable infinite subsets of $Y$, which are balls. Hence $Y$ is not locally finite.

Question 4.7. Does there exist a locally finite quasi-coarse space without a bounded geometry skeleton? 
Let $X$ be as in quasi-coarse space having a bounded geometry skeleton. We define $\mathrm{h}_{c}^{b g}(X)=\mathrm{h}_{c}\left(i d_{Y}\right)$, where $Y$ is a bounded geometry skeleton of $X$. Note that $\mathrm{h}_{c}^{b g}(X)$ is well-defined. In fact, if $Y$ and $Z$ are two bounded geometry skeletons of $X$, then $Y$ is Sym-coarsely equivalent to $Z$, and thus Corollary $4.1 \mathrm{implies} \mathrm{that}_{c}\left(i d_{Y}\right)=$ $\mathrm{h}_{c}\left(i d_{Z}\right)$.

Because of the monotonicity of the coarse entropy under taking invariant subspaces, $\mathrm{h}_{c}^{b g}(X) \leq \mathrm{h}_{c}\left(i d_{X}\right)$. Moreover, there exists a locally finite quasi-coarse space $X$ such that $\mathrm{h}_{c}^{b g}(X)<\mathrm{h}_{c}\left(i d_{X}\right)$. In order to find an example showing the strict inequality, we have to choose $X$ without bounded geometry. Let $X$ as in Example 2.3(a). Then Theorem 4.4 implies that $\mathrm{h}_{c}\left(i d_{X}\right)=\infty$. However, $Y=\mathbb{N} \times\{0\}$ is Sym-large in $X$ and $\mathrm{h}_{c}\left(i d_{Y}\right)=0$. Hence $\mathrm{h}_{c}^{b g}(X)=0$.

Let us recall the classical notion of growth type of non-decreasing functions from $\mathbb{N}$ to $\mathbb{R}_{\geq 0}$. Let $u, v: \mathbb{N} \rightarrow$ $\mathbb{R}_{\geq 0}$ be two non-decreasing functions. We say that $v$ dominates $u$, and we write $u \leq v$, if there are $a, b \geq 1$ and $c>0$ such that, for every $n \geq c, u(n) \leq a v(b n)$. Then $u$ and $v$ have the same growth type $(u \cong v)$ if $u \leq v \leq u$.

Let $u: \mathbb{N} \rightarrow \mathbb{R}_{\geq 0}$ be a non-decreasing function. Then the growth type of $u$ is:

- polynomial if $u$ is dominated by a polynomial function of some exact degree;

- sub-exponential if $u$ does not dominate any exponential function $n \mapsto a^{n}$;

- exponential if $u$ dominates an exponential function $n \mapsto a^{n}$.

We can characterise the previous classes of growth types as follows. If $u$ is a non-decreasing function, then

$$
\begin{array}{ccc}
u \text { has polynomial growth type if and only if } & \limsup _{n \rightarrow \infty} \frac{\log u(n)}{\log (n)}<\infty, \\
u \text { has sub-exponential growth type if and only if } & \limsup _{n \rightarrow \infty} \frac{\log u(n)}{n}=0, \quad \text { and } \\
u \text { has exponential growth type if and only if } & 0<\liminf _{n \rightarrow \infty} \frac{\log u(n)}{n}<\infty .
\end{array}
$$

Definition 4.8 ([2]). Let $(X, d)$ be a connected metric space having a bounded geometry skeleton. Define the growth rate of $X$ as the growth type of the function

$$
\gamma(n)=\left|B_{Y}(y, n)\right|, \quad \text { where } n \in \mathbb{N},
$$

for every bounded geometry skeleton $Y$ of $X$ and any point $y \in Y$.

It is proved in [2] that the growth rate of a metric space does not depend on the bounded geometry skeleton and on the point chosen.

We want to estimate the growth rate of a coarse space $X$ having a bounded geometry skeleton $Y$ which is monogenic. However, thanks to Lemma 1.6, the existence of such a $Y$ ensures that $X$ itself is monogenic. Hence we can consider monogenic quasi-coarse spaces having bounded geometry skeletons.

Theorem 4.9. Let $(X, d)$ be a metric space such that $\left(X, \mathcal{E}_{d}\right)$ is monogenic and connected, and has a bounded geometry skeleton $Y$. Let $\left.E \in \mathcal{E}\right|_{Y}$ be an entourage such that $\left\{E^{n} \mid n \in \mathbb{N}\right\}$ is a base of $\left.\mathcal{E}\right|_{Y}$, and $y \in Y$.

(a) If $X$ has polynomial growth type then $\mathrm{H}_{c}\left(i d_{Y}, y, E\right)=0$ and $\mathrm{h}_{c}^{b g}(X)=\mathrm{h}_{c}\left(i d_{Y}\right)=0$.

(b) X has sub-exponential growth type if and only if $\mathrm{H}_{c}\left(i d_{Y}, y, E\right)=0$ if and only if $\mathrm{h}_{c}^{b g}(X)=\mathrm{h}_{c}\left(i d_{Y}\right)=0$.

(c) X has exponential growth type if and only if $\mathrm{H}_{c}\left(i d_{Y}, y, E\right)>0$ if and only if $\mathrm{h}_{c}^{b g}(X)=\mathrm{h}_{c}\left(i d_{Y}\right)=\infty$.

Proof. Item (a) and the first equivalences of items (b) and (c) follow from (21). Corollary 4.5 and Proposition 2.2(b) imply the other two equivalences.

\section{Relationships with other entropies}

In this section we discuss the relationships of the coarse entropy with other well-known entropy notions in other branches of mathematics. In particular, we consider the coarse entropy of endomorphisms of groups 
and we connect it with the algebraic entropy (in §5.1) and, through the Pontryagin functor, with the topological and the measure entropy (in §5.2).

\subsection{Relationship with the algebraic entropy}

Let $M$ be a monoid. Let $f: M \rightarrow M$ be an endomorphism of $M$ (i.e., $f(x y)=f(x) f(y)$, for every $x, y \in M$, and $f(e)=e$, where $e$ is the neutral element of $M)$. Fix a finite subset $K \in[M]^{<\omega}$, and $n \in \mathbb{N} \backslash\{0\}$. Then the $n$-algebraic trajectory of $f$ with respect to $K$ is the subset

$$
\mathrm{T}_{n}^{a l g}(f, K)=K \cdot f(K) \cdots \cdots f^{n-1}(K) .
$$

Definition 5.1 ([10]). Let $M$ be a monoid, and $f: M \rightarrow M$ be an endomorphism of $M$. Then the algebraic entropy of $f$ with respect to $K$, where $K \in[M]^{<\omega}$, is defined as

$$
\mathrm{H}_{\text {alg }}(f, K)=\lim _{n \rightarrow \infty} \frac{\log \left|\mathrm{T}_{n}^{a l g}(f, K)\right|}{n} .
$$

Finally, the algebraic entropy of $f$ is $\mathrm{h}_{\text {alg }}(f)=\sup \left\{\mathrm{H}_{\text {alg }}(f, K) \mid K \in[M]^{<\omega}\right\}$.

A standard approach to prove that the limit in (22) exists is by using Fekete's Lemma (see, for example, [8]). We refer to [8] for a comprehensive survey on the algebraic entropy.

In this subsection, every monoid is endowed with its monoid-quasi-coarse structure. Since every monoid endomorphism is automatically bornologous, it is natural to compare its algebraic entropy with its coarse entropy.

Theorem 5.2. If $M$ is a monoid and $f: M \rightarrow M$ is an endomorphism, then

$$
\mathrm{h}_{c}(f) \leq \mathrm{h}_{\text {alg }}(f) \text {. }
$$

Moreover, if $f$ is surjective, then

$$
\mathrm{h}_{c}(f)=\mathrm{h}_{\text {alg }}(f) .
$$

Proof. Note that $\left\{E_{K} \mid K \in[M]^{<\omega}\right\}$ is cofinal in $\varepsilon_{M}$, and thus Proposition 2.2(a) implies that we just need to compute $\mathrm{H}_{c}^{l o c}(f, x)=\sup \left\{\mathrm{H}_{c}\left(f, x, E_{K}\right) \mid K \in[M]^{<\omega}\right\}$, for every $x \in M$. Moreover, since the identity $e$ is a maximum element (see Remark 1.5), Proposition 2.2(b) implies that $\mathrm{h}_{c}(f)=\mathrm{H}_{c}^{l o c}(f, e)$. For every $K \in[M]^{<\omega}$ and $n \in \mathbb{N} \backslash\{0\}$, according to (3), we have that

$$
\begin{aligned}
\mathrm{T}_{n}\left(f, e, E_{K}\right) & =\left(f^{n-1} \times f^{n-1}\right)\left(E_{K}\right)\left[\cdots\left[(f \times f)\left(E_{K}\right)\left[E_{K}[x]\right]\right] \cdots\right] \subseteq \\
& \subseteq E_{f^{n-1}(K)}\left[\cdots\left[E_{f(K)}\left[E_{K}[e]\right]\right] \cdots\right]=e K f(K) \cdots f^{n-1}(K)=\mathrm{T}_{n}^{a l g}(f, K) .
\end{aligned}
$$

Hence $\mathrm{H}_{c}\left(f, e, E_{K}\right) \leq \mathrm{H}_{a l g}(f, K)$ and thus

$$
\mathrm{h}_{c}(f)=\mathrm{H}_{c}^{l o c}(f, e)=\sup \left\{\mathrm{H}_{c}\left(f, e, E_{K}\right) \mid K \in[M]^{<\omega}\right\} \leq \sup \left\{\mathrm{H}_{a l g}(f, K) \mid K \in[M]^{<\omega}\right\}=\mathrm{h}_{\text {alg }}(f) .
$$

Moreover, if $f$ is surjective, $(f \times f)\left(E_{K}\right)=E_{f(K)}$, for every $K \in[M]^{<\omega}$, and thus the inclusion in (23) becomes an equality, which proves the desired formula.

Thanks to Theorem 5.2, we can reinterpret what we have obtained so far as generalisations, in the case of a surjective homomorphism of monoids, of classical results in the realm of the algebraic entropy. Results that can be seen from this point of view are Proposition 2.2(a), Remark 2.5(c), Proposition 3.3, Theorem 3.5(a), Corollary 3.9, Theorem 4.4, and Theorem 4.9 in the case of finitely generated groups (see [8] for a comprehensive survey on algebraic entropy containing the mentioned results).

Let $M$ be a monoid and $g: M \rightarrow M$ be an endomorphism. We want to specialise formula (7) in this setting. Let $K$ be a finite subset of $M$. Then

$$
(g \times g)\left(E_{K}\right)[A]=g\left(E_{K}\left[g^{-1}(A)\right]\right)=g\left(g^{-1}(A) K\right)=(A \cap g(M)) g(K) .
$$

The inequality in Theorem 5.2 may be strict, as Examples 5.3 and 5.4 show. 
Example 5.3. Let $G$ be a finitely generated group endowed with one of its word metrics. Consider the homomorphism $f: \bigoplus_{n \in \mathbb{N}} G \rightarrow \bigoplus_{n \in \mathbb{N}} G$, called the right Bernoulli shift, such that $f\left(x_{0}, x_{1}, x_{2}, \ldots\right)=$ $\left(0, x_{0}, x_{1}, \ldots\right)$, for every $\left(x_{n}\right)_{n} \in \bigoplus_{n} G$. We claim that $\mathrm{h}_{c}(f)=0$, while, for example, if $G=\mathbb{Z}_{m}$, the finite cyclic group with $m$ elements, then $\mathrm{h}_{\text {alg }}(f)=\log m$.

For every $(i, m, n) \in \mathbb{N}^{3}$, define the subset

$$
A(i, m, n)=\underbrace{\{0\} \oplus \cdots \oplus\{0\}}_{i \text { times }} \oplus \underbrace{B(e, m) \oplus \cdots \oplus B(e, m)}_{n \text { times }} \oplus\{0\} \oplus \cdots .
$$

Note that $\left\{E_{A(0, m, m)}\right\}_{m \in \mathbb{N}}$ forms a base of the coarse structure $\varepsilon_{\oplus_{n} G}$ and thus Proposition 2.2 implies that, in order to compute the coarse entropy, we can restrict ourselves to just consider the values $\mathrm{H}_{c}\left(f, 0, E_{A(0, m, m)}\right)$, for every $m \in \mathbb{N}$. Note that, if $m \in \mathbb{N}, \mathrm{T}_{1}\left(f, 0, E_{A(0, m, m)}\right)=A(0, m, m)$, and, moreover, we claim that, for every $0 \leq n \leq m$,

$$
\mathrm{T}_{n+1}\left(f, 0, E_{A(0, m, m)}\right)=\prod_{i=m-n}^{m} A(n, m, i) .
$$

Suppose that, for some $0 \leq n<m$, (25) holds. Then, according to (24),

$$
\begin{aligned}
\mathrm{T}_{n+2}\left(f, 0, E_{A(0, m, m)}\right) & =\left(\mathrm{T}_{n+1}\left(f, 0, E_{A(0, m, m)}\right) \cap f^{n+1}\left(\bigoplus_{n \in \mathbb{N}} G\right)\right) f^{n+1}(A(0, m, m))= \\
& =\left(\left(\prod_{i=m-n}^{m} A(n, m, i)\right) \cap f^{n+1}\left(\bigoplus_{n \in \mathbb{N}} G\right)\right) f^{n+1}(A(0, m, m))= \\
& =\left(\prod_{i=m-n}^{m} A(n+1, m, i-1)\right) A(n+1, m, m)=\prod_{i=m-(n+1)}^{m} A(n+1, m, i) .
\end{aligned}
$$

Hence, we have proved (25), for every $0 \leq n \leq m$. By substituting $n=m$, we have

$$
\mathrm{T}_{m+1}\left(f, 0, E_{A(0, m, m)}\right)=\prod_{i=0}^{m} A(m, m, i) .
$$

Applying again (24), we can show that, for every $k \in \mathbb{N}$,

$$
\mathrm{T}_{m+k+1}\left(f, 0, E_{A(0, m, m)}\right)=\prod_{i=0}^{m} A(m+k, m, i),
$$

and so $\left|\mathrm{T}_{m+1}\left(f, 0, E_{A(0, m, m)}\right)\right|=\left|\mathrm{T}_{m+k+1}\left(f, 0, E_{A(0, m, m)}\right)\right|$. Since the cardinality of the trajectories stabilises, $\mathrm{H}_{c}\left(f, 0, E_{A(0, m, m)}\right)=0$, for every $m \in \mathbb{N}$, and thus $\mathrm{h}_{c}(f)=0$.

Example 5.4. Let $G=\mathbb{Z}$, and, for $k \in \mathbb{N} \backslash\{0\}$, define the endomorphism $f=f_{k}: x \mapsto k x$ of $G$. We claim that $\mathrm{h}_{c}(f)=0$, while $\mathrm{h}_{\text {alg }}(f)=\log k$.

The case $k=1$ easily follows, since $f_{1}=i d_{\mathbb{Z}}$ and $\mathrm{h}_{c}\left(f_{1}\right)=\mathrm{h}_{\text {alg }}\left(f_{1}\right)=0$ according to Theorem 5.2, so we can assume that $k>1$. Since $G$ is connected, we can consider the trajectories centred at 0 (Proposition 2.2(b)). Moreover, the family $\left\{E_{[-m, m]}\right\}_{m \in \mathbb{N}}$ forms a base of the coarse structure $\mathcal{E}_{G}$ and thus we can restrict ourselves to considering only those induced entourages (Proposition 2.2(a)). According to (23) and the definition of algebraic trajectories, for every $n \in \mathbb{N}$,

$$
\mathrm{T}_{n}\left(f, 0, E_{[-m, m]}\right) \subseteq \mathrm{T}_{n}^{a l g}(f,[-m, m])=\left[-m\left(\sum_{i=0}^{n-1} k^{i}\right), m\left(\sum_{i=0}^{n-1} k^{i}\right)\right] .
$$

Hence, (26) and (24) imply that, for every $n \in \mathbb{N}$,

$$
\mathrm{T}_{n+1}\left(f, 0, E_{[-m, m]}\right) \subseteq\left(\mathrm{T}_{n}^{a l g}(f,[-m, m]) \cap f^{n}(G)\right)+f^{n}([-m, m]),
$$


and thus

$$
\begin{aligned}
\left|\mathrm{T}_{n+1}\left(f, 0, E_{[-m, m]}\right)\right| & \leq\left|\left[-m\left(\sum_{i=0}^{n-1} k^{i}\right), m\left(\sum_{i=0}^{n-1} k^{i}\right)\right] \cap f^{n}(M)\right| \cdot(2 m+1) \leq \\
& \leq\left\lceil 1+\frac{1}{k^{n}} 2 m \sum_{i=0}^{n-1} k^{i} \mid(2 m+1) \leq(2 m+2)^{2} .\right.
\end{aligned}
$$

Since the trajectories have bounded cardinality, $\mathrm{H}_{c}\left(f, 0, E_{[-m, m]}\right)=0$ and thus $\mathrm{h}_{c}(f)=0$.

Furthermore, if we consider the group $H=\mathbb{Q}$ and the endomorphism $f=f_{k}: H \rightarrow H$, since $f$ is surjective, $\mathrm{h}_{c}(f)=\mathrm{h}_{a l g}(f)=\log k$ (Theorem 5.2). Hence, there is no Addition Theorem for the coarse entropy. In fact, consider the following diagram

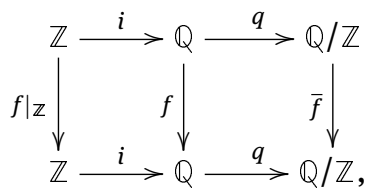

where $i$ and $q$ are the canonical inclusion and quotient, respectively, and $\left.f\right|_{\mathbb{Z}}$ is the restriction of $f$ to $\mathbb{Z}$ and $\bar{f}$ is the projection of $f$. While $\mathrm{h}_{\text {alg }}(f)=\log k=\mathrm{h}_{\text {alg }}\left(\left.f\right|_{\mathbb{Z}}\right)+\mathrm{h}_{\text {alg }}(\bar{f})$ (an instance of the Addition Theorem for the algebraic entropy, see for example [10]), $\mathrm{h}_{c}(f)=\log k$, while $\mathrm{h}_{c}\left(\left.f\right|_{\mathbb{Z}}\right)=0$ and $\mathrm{h}_{c}(\bar{f})=\mathrm{h}_{\text {alg }}(\bar{f})=0$, since $\bar{f}$ is surjective, and thus $\mathrm{h}_{c}(f) \neq \mathrm{h}_{c}\left(\left.f\right|_{\mathbb{Z}}\right)+\mathrm{h}_{c}(\bar{f})$.

Example 5.5. Let $F_{2}$ be the free group freely generated by two letters $a$ and $b$. Consider the injective endomorphism $f: F_{2} \rightarrow F_{2}$ such that $f(a)=a^{2}$ and $f(b)=b^{2}$. We claim that $\mathrm{h}_{c}(f)=0$.

Thanks to Proposition 2.2, we can just consider the trajectories with respect to $e$ and $E_{B(e, m)}$, where $m \in \mathbb{N}$ and $F_{2}$ is endowed with the word metric associated with the standard generating set $\left\{a, b, a^{-1}, b^{-1}\right\}$. Let us inductively define a sequence of natural numbers $\left\{g_{n}\right\}_{n}$ as follows:

$$
\left\{\begin{array}{l}
g_{0}=0, \\
g_{n+1}=m+\left\lfloor g_{n} / 2\right\rfloor .
\end{array}\right.
$$

By induction, it is easy to see that the sequence $\left\{g_{n}\right\}_{n}$ is bounded by $2 m$.

We claim that, for every $n \in \mathbb{N}$,

$$
\mathrm{T}_{n+1}\left(f, e, E_{B(e, m)}\right)=f^{n}\left(B\left(e, g_{n+1}\right)\right) .
$$

If $n=0$, then (27) is satisfied. Suppose that for some $n \in \mathbb{N}$, (27) holds. Then, applying (24),

$$
\begin{aligned}
\mathrm{T}_{n+2}\left(f, e, E_{B(e, m)}\right) & =\left(\left(f^{n+1} \times f^{n+1}\right)\left(E_{B(e, m)}\right)\right)\left[\mathrm{T}_{n+1}\left(f, e, E_{B(e, m)}\right)\right]= \\
& =f^{n+1}\left(f^{-n-1}\left(f^{n}\left(B\left(e, g_{n+1}\right)\right)\right) B(e, m)\right)= \\
& =f^{n+1}\left(B\left(e,\left\lfloor g_{n+1} / 2\right\rfloor\right) B(e, m)\right)=f^{n+1}\left(B\left(e, g_{n+2}\right)\right) .
\end{aligned}
$$

Finally, (27) implies that

$$
\mathrm{H}_{c}\left(f, e, E_{B(0, m)}\right)=\limsup _{n \rightarrow \infty} \frac{\log \left(\left|B\left(e, g_{n+1}\right)\right|\right)}{n+1} \leq \limsup _{n \rightarrow \infty} \frac{g_{n+1} \log 5}{n+1}=0
$$

since $g_{n+1} \leq 2 m$. Hence, $\mathrm{h}_{c}(f)=0$.

In Examples 5.3, 5.4, and 5.5 we provided endomorphisms of groups $f: G \rightarrow G$ such that $\bigcap_{n} f^{n}(G)=\{e\}$ and $\mathrm{h}_{c}(f)=0$. Hence the following question naturally arises.

Question 5.6. Let $f: M \rightarrow M$ be a endomorphism of a monoid $M$ such that $\bigcap_{n} f^{n}(M)$ is finite. Is it true that $\mathrm{h}_{c}(f)=0$ ?

Note that, in the notation of Example 2.6(c), even though $\bigcap_{n} f^{n}(M)=\emptyset, \mathrm{h}_{c}(f)=\infty$, but $f$ is not an endomorphism. 


\subsection{Relationship with the topological and the measure entropy}

In this subsection we want to relate the coarse entropy with the topological entropy and the measure entropy, using the Pontryagin duality.

Let $\vartheta_{\tau}\left(0_{\mathbb{T}}\right)$ be the family of all open neighbourhoods of the identity of the torus $\mathbb{T}$. Consider a topological abelian group $G$, and denote by $\widehat{G}$ the family of all continuous characters $\chi: G \rightarrow \mathbb{T}$. With the pointwise operation, $\widehat{G}$ is actually an abelian group. We can endow $\widehat{G}$ with the compact-open topology $\widehat{\tau}$, which is defined by the following base of neighbourhoods of the null-character $0 \in \widehat{G}$ :

$$
\left\{W_{\widehat{G}}(K, U) \mid K \subseteq G \text { compact, } U \in \vartheta_{\tau}\left(0_{\mathbb{T}}\right)\right\}, \quad \text { where, }
$$

for every compact subset $K \subseteq G$ and every $U \in \vartheta_{\tau}\left(0_{\mathbb{T}}\right), \quad W_{\widehat{G}}(K, U)=\{\chi \in \widehat{G} \mid \chi(K) \subseteq U\}$.

With this topology, $\widehat{G}$ is a topological abelian group, called the dual group of $G$. For every continuous homomorphism $f: G \rightarrow H$ between topological abelian groups, there exists a continuous homomorphism $\widehat{f}: \widehat{H} \rightarrow \widehat{G}$ defined by the law $\widehat{f}(\chi)=\chi \circ f$, for every $\chi \in \widehat{H}$. The Pontryagin-van Kampen duality theorem states that the functor $\widehat{\bullet}: \mathcal{L} \rightarrow \mathcal{L}$, where $\mathcal{L}$ is the category of locally compact groups, induces a duality (see [33] for details). In particular, recall that, a locally compact abelian group $G$ is discrete if and only if $\widehat{G}$ is compact.

The Pontryagin duality is a powerful tool to translate concepts and results from the small-scale geometry to the large-scale geometry of locally compact abelian groups. In particular, we cite a result due to Nicas and Rosenthal ([28]): for every locally compact abelian group $G$, the covering dimension of $G([18])$ is equal to the asymptotic dimension (the large-scale counterpart of the covering dimension, see, for example, $[3,29]$ ) of $\widehat{G}$, where $\widehat{G}$ is endowed with its compact-group-coarse structure. Other examples of those connections can be found in [14]. Let us just recall the following result.

Proposition $5.7([15])$. Let $G$ be a locally compact abelian group. Then $\left(G, \mathcal{E}_{\mathfrak{r} \mathfrak{C}(G)}\right)$ is metrisable (i.e., there exists a metric $d$ on $G$ such that $\left.\mathcal{E}_{\mathfrak{r} \mathfrak{C}(G)}=\mathcal{E}_{d}\right)$ if and only if $\widehat{G}$ is metrisable as a topological group.

In the realm of entropies, the Pontryagin duality plays an important role since it connects the algebraic entropy with the topological entropy (see Theorem 5.9).

Let $f: X \rightarrow X$ be a self-map of a set $X$ and $\mathcal{U}$ a cover of $X$. Then we denote by $f^{-1}(\mathcal{U})=\left\{f^{-1}(U) \mid U \in \mathcal{U}\right\}$. Moreover, if $\mathcal{V}$ is another cover of $X$, then we define $U \vee \mathcal{V}=\{U \cap V \mid U \in \mathcal{U}, V \in \mathcal{V}\}$.

Let now $X$ be a compact space. Denote by $\operatorname{cov}(X)$ the family of all open covers of $X$. For every cover $U \in$ $\operatorname{cov}(X)$, denote by $N(\mathcal{U})$ the minimum of the cardinalities of finite subcovers of $\mathcal{U}$. For every $\mathcal{U} \in \operatorname{cov}(X)$, define the entropy of $U$ as $\mathrm{H}(\mathcal{U})=\log N(\mathcal{U})$.

Definition $5.8([1])$. Let $f: X \rightarrow X$ be a continuous self-map of a compact space. For $\mathcal{U} \in \operatorname{cov}(X)$, define the topological entropy of $f$ with respect to $U$ as

$$
\mathrm{H}_{\text {top }}(f, \mathcal{U})=\lim _{n \rightarrow \infty} \frac{\mathrm{H}\left(\mathcal{U} \vee f^{-1}(\mathcal{U}) \vee \cdots \vee f^{-n+1}(\mathcal{U})\right)}{n} .
$$

Then the topological entropy of $f$ is

$$
\mathrm{h}_{\text {top }}(f)=\sup \left\{\mathrm{H}_{\text {top }}(f, \mathcal{U}) \mid \mathcal{U} \in \operatorname{cov}(X)\right\} .
$$

Theorem 5.9 (Bridge theorem, [9]). Let $f: G \rightarrow G$ be an endomorphism of a group $G$. Then

$$
\mathrm{h}_{\text {alg }}(f)=\mathrm{h}_{\text {top }}(\widehat{f}) .
$$

By combining Theorems 5.2 and 5.9 we obtain the following result.

Corollary 5.10. Let $f: G \rightarrow G$ be an endomorphism of a group $G$. Then

$$
\mathrm{h}_{c}(f) \leq \mathrm{h}_{\text {alg }}(f)=\mathrm{h}_{\text {top }}(\widehat{f}) .
$$


Moreover, if $f$ is surjective, then

$$
\mathrm{h}_{c}(f)=\mathrm{h}_{\text {alg }}(f)=\mathrm{h}_{\text {top }}(\widehat{f})
$$

Before ending the section, let us connect the coarse entropy also with the measure entropy. Let $(X, \mathfrak{B}, \mu)$ be a measure space and $\xi=\left\{A_{i} \mid i=1, \ldots, n\right\}$ be a measurable partition of $X$. Define the entropy of $\xi$ by $\mathrm{H}(\xi)=-\sum_{i=1}^{n} \mu\left(A_{i}\right) \log \left(\mu\left(A_{i}\right)\right)$.

Definition $5.11([25,40])$. Let $X$ be a measure space and $f: X \rightarrow X$ be a measure preserving map. If $\xi$ is a measurable partition of $X$, the measure entropy of $f$ with respect to $\xi$ is

$$
\mathrm{H}_{\text {mes }}(f, \xi)=\lim _{n \rightarrow \infty} \frac{H\left(\xi \vee f^{-1}(\xi) \vee \cdots \vee f^{-n+1}(\xi)\right)}{n} .
$$

The measure entropy of $f$ is

$$
\mathrm{h}_{m e s}(f)=\sup \left\{\mathrm{H}_{m e s}(f, \xi) \mid \xi \text { measurable partition of } X\right\} .
$$

In the realm of compact groups, the following result was proved by Stoyanov.

Theorem 5.12 ([41]). Let $G$ be a compact metrisable group and $f: G \rightarrow G$ be a continuous surjective homomorphism. If $G$ is endowed with its Haar measure, then

$$
\mathrm{h}_{\text {top }}(f)=\mathrm{h}_{\text {mes }}(f) .
$$

Corollary 5.13. Let $f: G \rightarrow G$ be an automorphism of a countable group $G$. Then

$$
\mathrm{h}_{c}(f)=\mathrm{h}_{\text {alg }}(f)=\mathrm{h}_{\text {top }}(\widehat{f})=\mathrm{h}_{\text {mes }}(\widehat{f}) .
$$

Proof. Corollary 5.10 states the first two equalities. Since $G$ is countable and discrete, $\left(G, \varepsilon_{\mathfrak{r e}(G)}\right)=\left(G, \varepsilon_{G}\right)$ is metrisable ([16]). Hence Proposition 5.7 implies that $\widehat{G}$ is metrisable as topological group. Moreover, since $f$ is an isomorphism, also $\widehat{f}$ is an isomorphism and so we can apply Theorem 5.12 to get the claim.

Acknowledgements: It is a pleasure to thank the referee for the careful reading and valuable suggestions that greatly improved the paper.

The author was partially supported by the Project SIR2014 - GADYGR.

\section{References}

[1] R. L. Adler, A. G. Konheim, M. H. McAndrew, Topological entropy, Trans. Amer. Math. 114 (1965) 309-319.

[2] J. A. Álvarez López, A. Candel, Generic coarse geometry of leaves, Lecture Notes in Mathematics, Springer International Publishing (2018).

[3] G. Bell, A. N. Dranishnikov, Asymptotic dimension. Topology Appl. 155 (2008), 1265-1296.

[4] R. Bowen, Entropy for group endomorphisms and homogeneous spaces, Trans. Amer. Math. Soc. 153 (1971) $401-414$.

[5] T. Ceccherini-Silberstein, M. Coornaert, F. Krieger, An analogue of Fekete's lemma for subadditive functions on cancellative amenable semigroups, J. Analyse Math. 124 (2014) 59-81.

[6] D. Dikranjan, A. Fornasiero, A. Giordano Bruno, Algebraic entropy for amenable semigroup actions, preprint.

[7] D. Dikranjan, A. Giordano Bruno, Functorial topologies, Topology Appl. 158 (17) (2011), 2261-2442.

[8] D. Dikranjan, A. Giordano Bruno, Topological entropy and algebraic entropy for group endomorphisms, Proceedings ICTA2011 Islamabad, Pakistan July 4-10 2011 Cambridge Scientific Publishers 133-214.

[9] D. Dikranjan, A. Giordano Bruno, The connection betwwen topological and algebraic entropy, Topology Appl. 159 (13) (2012) 2980-2989.

[10] D. Dikranjan, A. Giordano Bruno, Entropy on abelian groups, Advances in Mathematics 298 (2016), 612-653.

[11] D. Dikranjan, A. Giordano Bruno, Entropy on normed semigroups (Towards a unifying approach to entropy), Dissertationes Math. 542 (2019), 1-90.

[12] D. Dikranjan, B. Goldsmith, L. Salce, P. Zanardo, Algebraic entropy for abelian groups, Trans. Amer. Math. Soc. 361 (2009), 3401-3434. 
[13] D. Dikranjan, N. Zava, Some categorical aspects of coarse spaces and balleans, Topology Appl., 225 (2017), 164-194.

[14] D. Dikranjan, N. Zava, Categories of coarse groups: quasi-homomorphisms and functorial coarse structures, Topology Appl., to appear.

[15] D. Dikranjan, N. Zava, The impact of Pontryagin and Bohr functors on large-scale properties of LCA groups, Topology Appl., to appear.

[16] A. Dranishnikov, J. Smith, Asymptotic dimension of discrete groups. Fund. Math. 189 (1) (2006) 27-34.

[17] J. Dydak, C. S. Hoffland, An alternative definition of coarse structures, Topology Appl. 155 (2008), no. 9, $1013-1021$.

[18] R. Engelking, Dimension Theory. North Holland Publishing Company, 1978.

[19] M. Gromov, Asymptotic invariants of infinite groups, Geometric group theory, vol. 2 (Sussex, 1991), 1-295, London Math. Soc. Lecture Notes Ser., 182, Cambridge Univ. Press, Cambridge, 1993.

[20] P. de la Harpe, Topics in geometric group theory. Chicago Lectures in Math., the University of Chicago Press, Chicago (2000).

[21] K.H. Hofmann, L. Stoyanov, Topological entropy of group and semigroup actions, Adv. Math. 115 (1995) no. 1, 54-98.

[22] B. M. Hood, Topological entropy and uniform spaces, J. London Math. Soc. 8 (2) (1974) 235-242.

[23] J. R. Isbell, Uniform Spaces, American Mathematical Society (1964).

[24] P. Fletcher, W. F. Lindgren, Quasi-Uniform Spaces, Lecture Notes Pure Appl. Math., 77, Dekker, New York (1982).

[25] A. N. Kolmogorov, New metric invariants of transitive dynamical systems and automorphisms of Lebesgue spaces, Doklady Akad. Nauk. SSSR 119 (1958) 861-864.

[26] H.-P. Kunzi, Nonsymmetric distances and their associated topologies: about the origins of basic ideals in the area of asymmetric topology, in Handbook of the history of general topology, Vol. 3, 853-968, Hist. Topol., 3, Kluwer Acad. Publ., Dordrecht, 2001.

[27] A. Nicas, D. Rosenthal, Coarse structures on groups, Topology Appl. 159 (14) (2012) 3215-3228.

[28] A. Nicas, D. Rosenthal, On the asymptotic dimension of the dual group of a locally compact abelian group, Topology Appl. 160 (2013), 682-684.

[29] P. W. Nowak, G. Yu, Large Scale Geometry. European Mathematical Society, 2012.

[30] D. Ornstein, B. Weiss, Entropy and isomorphism theorems for actions of amenable groups, J. Analyse Math. 48 (1987) 1-141.

[31] J. Peters, Entropy on discrete abelian groups, Adv. Math. 33 (1979), 1-13.

[32] J. Peters, Entropy of automorphisms on L.C.A. groups, Pacific J. Math. 96 (2) (1981) 475-488.

[33] L. S. Pontryagin, Topological Groups. Gordon and Breach, Science Publishers, Inc., 1966.

[34] I. V. Protasov, Asymptotic proximities, Applied Gen. Top., 9 (2008), no. 2, 189-195.

[35] I. V. Protasov, T. Banakh, Ball Structures and Colorings of Groups and Graphs, Math. Stud. Monogr. Ser., vol. 11, VNTL, Lviv, 2003.

[36] I. V. Protasov, O. I. Protasova, Sketch of group balleans, Matematychni Studii, 22 (2004), no. 1, 10-20.

[37] I. V. Protasov, M. Zarichnyi, General Asymptology, 2007 VNTL Publishers, Lviv, Ukraine.

[38] J. Roe, Lectures on Coarse Geometry, Univ. Lecture Ser., vol. 31, American Mathematical Society, Providence RI, 2003.

[39] C. E. Shannon, A Mathematical Theory of Communication, Bell System Technical Journal, 27 (3) (1948) 379-423.

[40] Y. G. Sinai, On the concept of entropy of a dynamical system, Doklady Akad. Nauk. SSSR 124 (1959) 789-781 (in Russian).

[41] L. N. Stoyanov, Uniqueness of topological entropy for endomorphisms on compact groups, Boll. Un. Mat. Ital. 1 (3) (1987) 829-847.

[42] S. Virili, Entropy for endomorphisms of LCA groups, Topology Appl. 159 (9) (2012), 2546-2556.

[43] M. D. Weiss, Algebraic and other entropies of group endomorphisms, Math. Systems Theory 8 (3) (1974/75) 243-248.

[44] N. Zava, Generalisations of coarse spaces, Topology Appl. 263 (2019), 230-256.

[45] N. Zava, Cowellpoweredness and closure operators in categories of coarse spaces, Topology Appl. 268 (2019), 106899. 